\title{
Calculation of the Zeeman effect for transition-metal complexes by multiconfiguration pair-density functional theory
}

\author{
Chen Zhou, ${ }^{\&, a}$ Dihua Wu, ${ }^{\&, a}$ Laura Gagliardi, ${ }^{*, b}$ and Donald G. Truhlar ${ }^{*, a}$ \\ ORCID \\ Chen Zhou: 0000-0002-6332-4198 \\ Dihua Wu: 0000-0001-8508-8723 \\ Laura Gagliardi: 0000-0001-5227-1396 \\ Donald G. Truhlar: 0000-0002-7742-7294
}

\footnotetext{
${ }^{\&}$ These two authors contributed equally to this paper.

${ }^{a}$ Department of Chemistry, Chemical Theory Center, and Minnesota Supercomputing Institute, University of Minnesota, Minneapolis, Minnesota 55455-0431, United States.

${ }^{b}$ Department of Chemistry, Pritzker School of Molecular Engineering, The James Franck Institute and Chicago Center for Theoretical Chemistry, The University of Chicago, Chicago, IL 60637, United States

*corresponding authors: lgagliardi@uchicago.edu,truhlar@umn.edu
}

\section{TABLE OF CONTENTS}

Section 1. Input example: MC-PDFT for $\left[\mathrm{Cu}\left(\mathrm{NH}_{3}\right)_{4}\right]^{2+}$ molecule 
Section S1. Input example: MC-PDFT calculation for $\left[\mathrm{Cu}\left(\mathrm{NH}_{3}\right)_{4}\right]^{2+}$ molecule

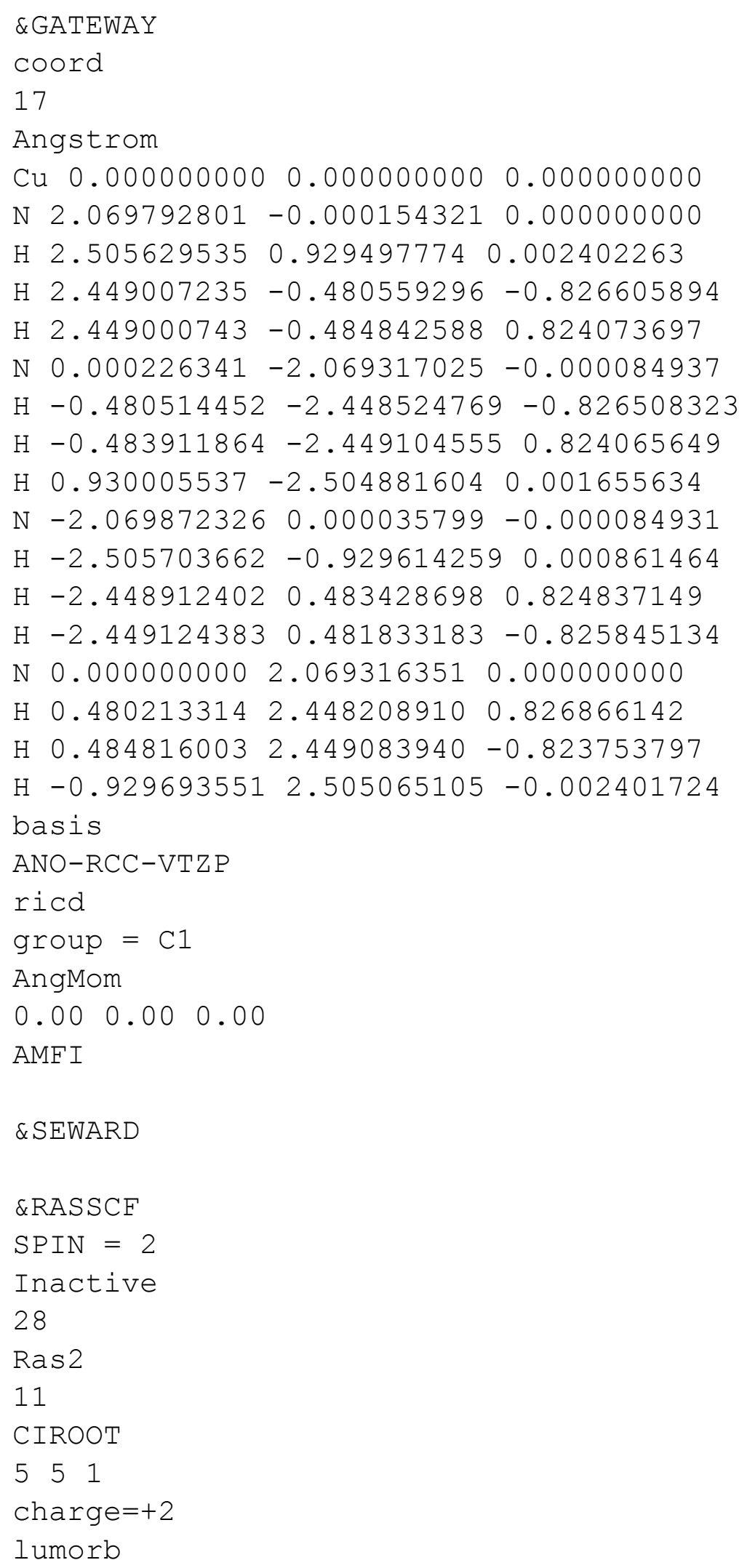




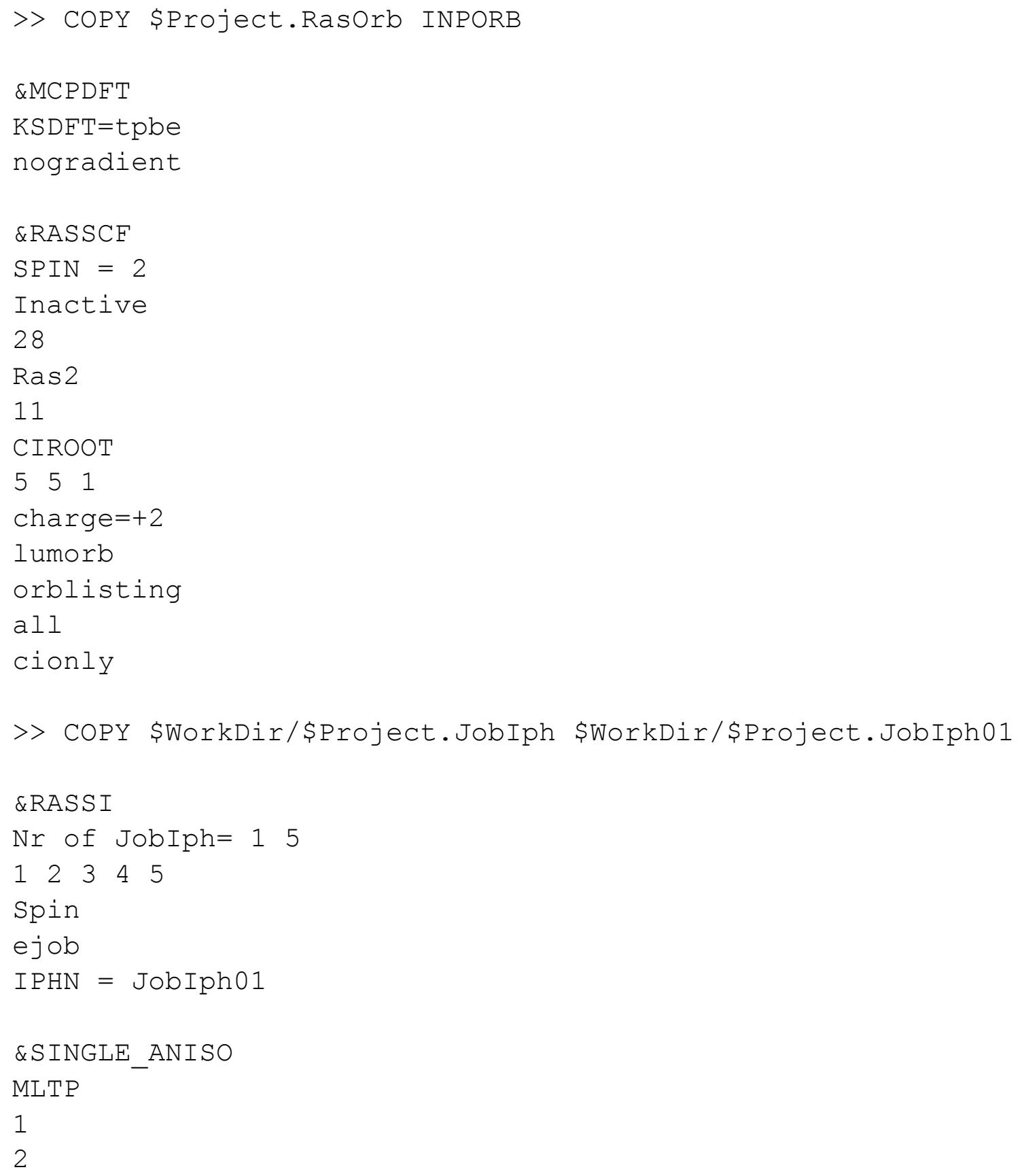


Section S2. Input example: XMS-PDFT calculation for $\left[\mathrm{Cu}\left(\mathrm{NH}_{3}\right)_{4}\right]^{2+}$ molecule. Note that for the following input file, the phase of the wave functions for the two RASSCF calculations should be the same for the XMS transformation. In the current version of locally modified codes, the phases of the states during the RASSCF calculation are chosen so that the largest CI coefficient of each state is positive. When we copy the orbitals of the first RASSCF calculation to be the initial guess for the second RASSCF calculation, and use "cionly" keyword in the second RASSCF calculation to keep the phases of the orbitals unchanged. In the future we will have a new version of the code that needs only one RASSCF calculation to avoid the phase problem of the current version, but that new version was not yet available when the present calculations were done.

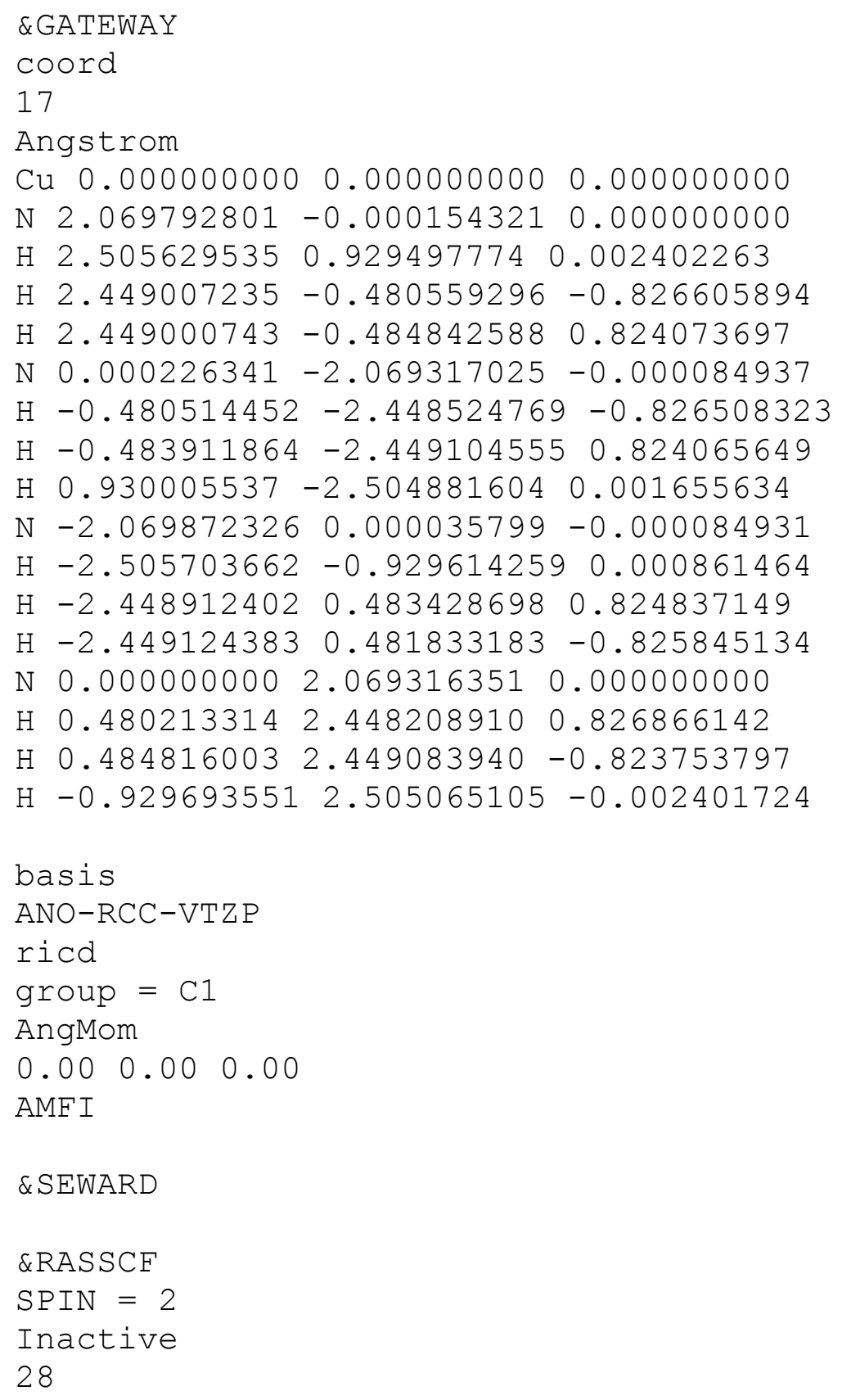




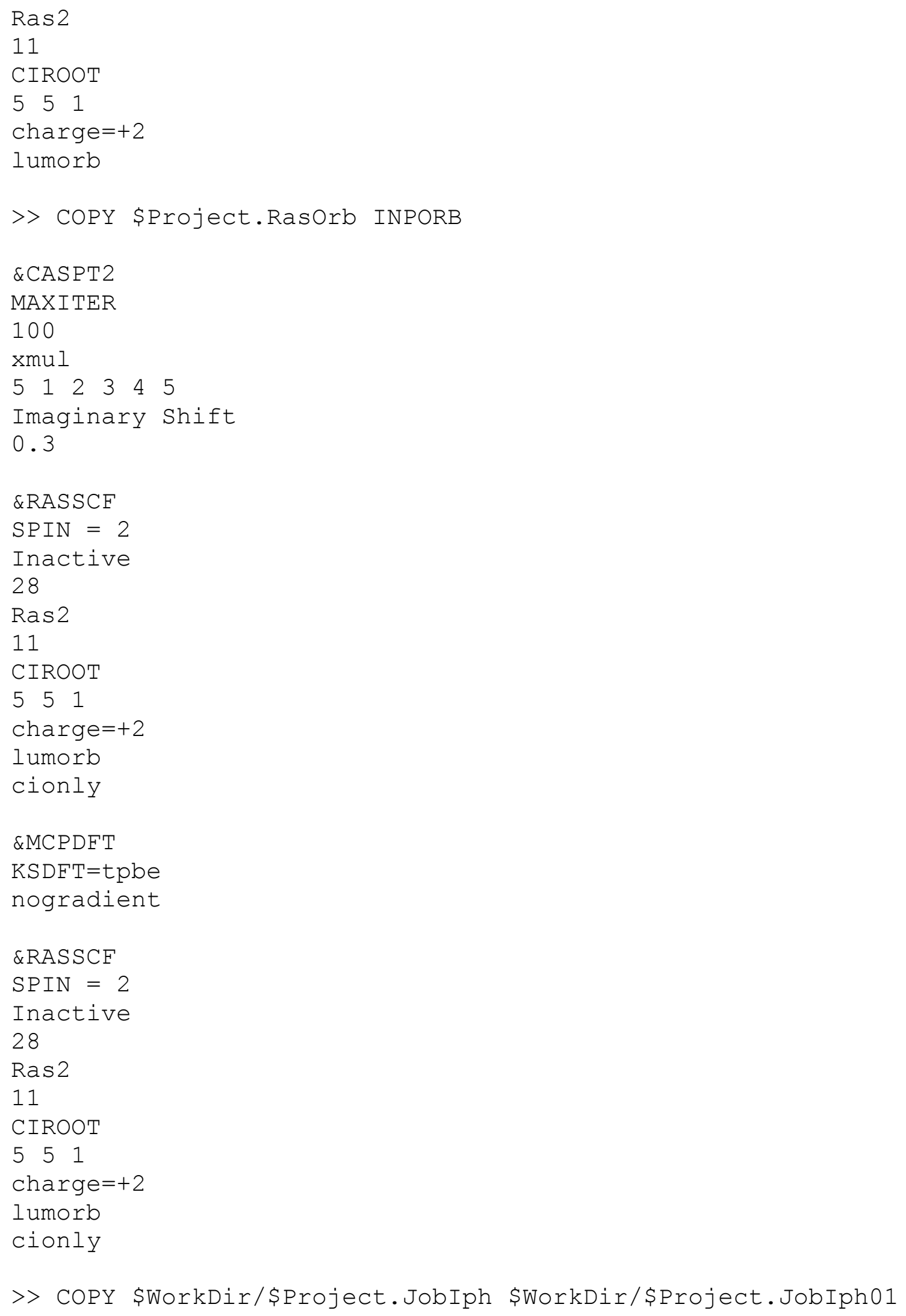


$\&$ RASS I

$\mathrm{Nr}$ of JobIph= 15

$\begin{array}{lllll}1 & 2 & 3 & 4 & 5\end{array}$

Spin

ejob

IPHN = JobIph01

$£$ SINGLE_ANISO

MLTP

1

2 
Section S3. Input example: MC(1,10)-PDFT calculation for [Co(sacsac $\left.)_{2}\right]$ molecule.

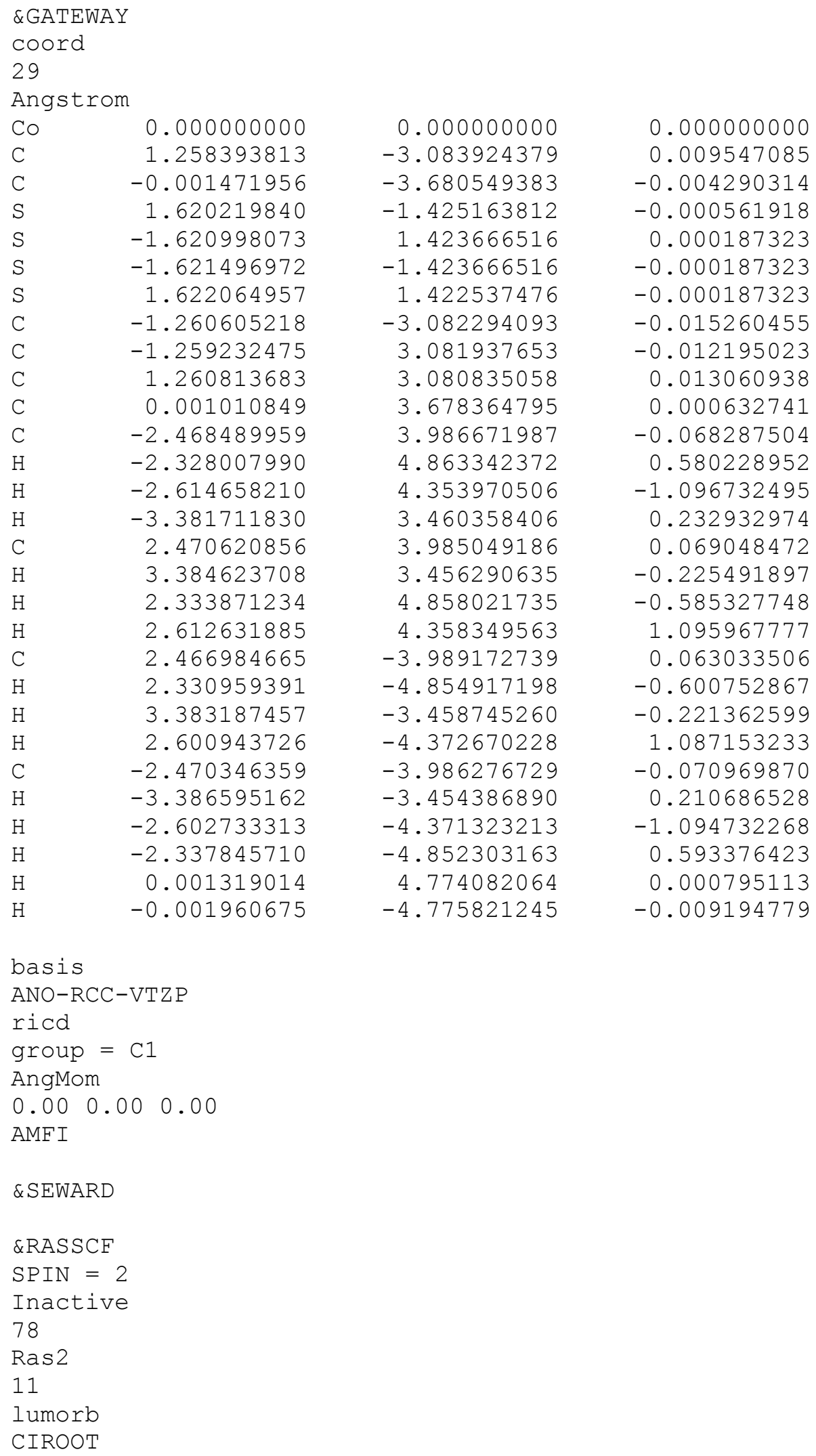




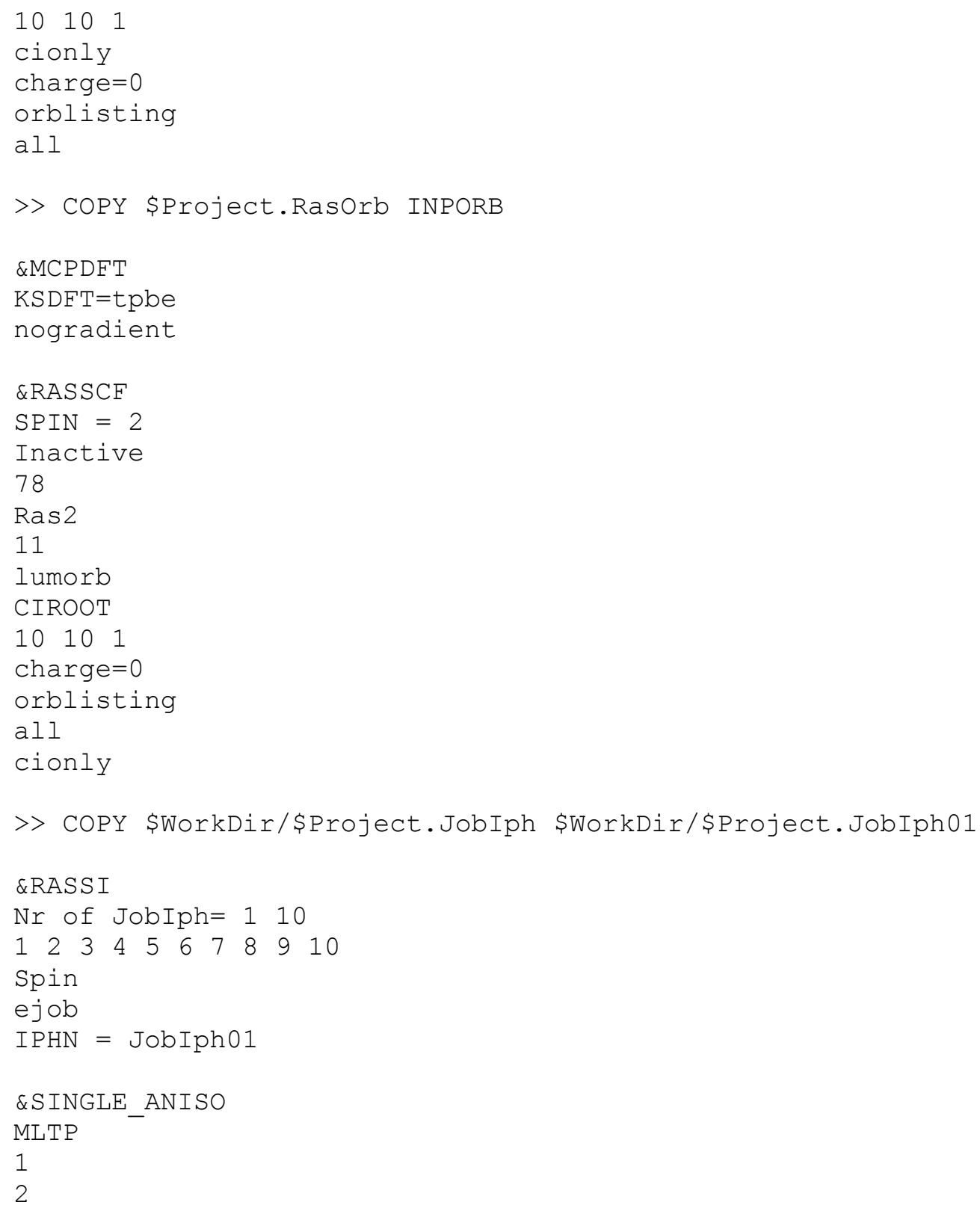


Section S4. Input example: XMS(3,10)-PDFT calculation for $\left[\mathrm{Fe}(\mathrm{bpy})_{3}\right]^{3+}$ molecule.

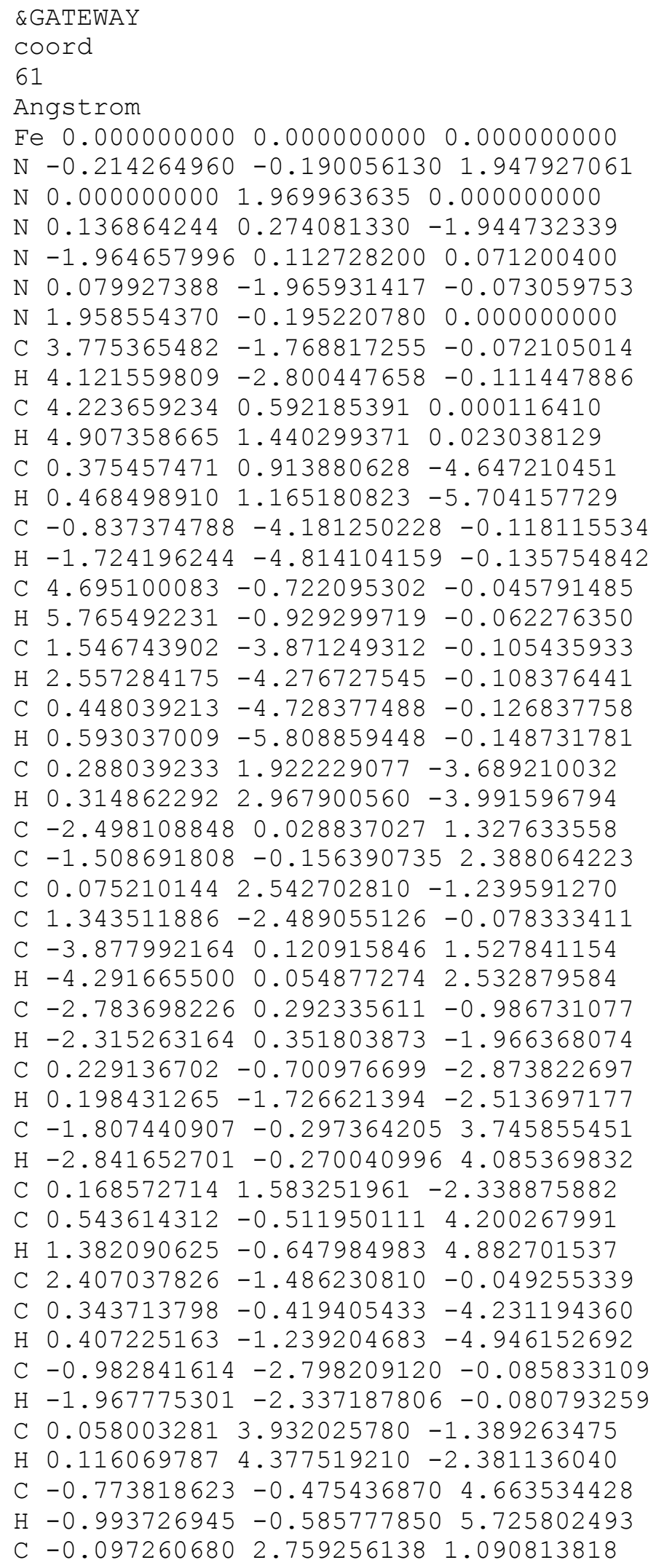




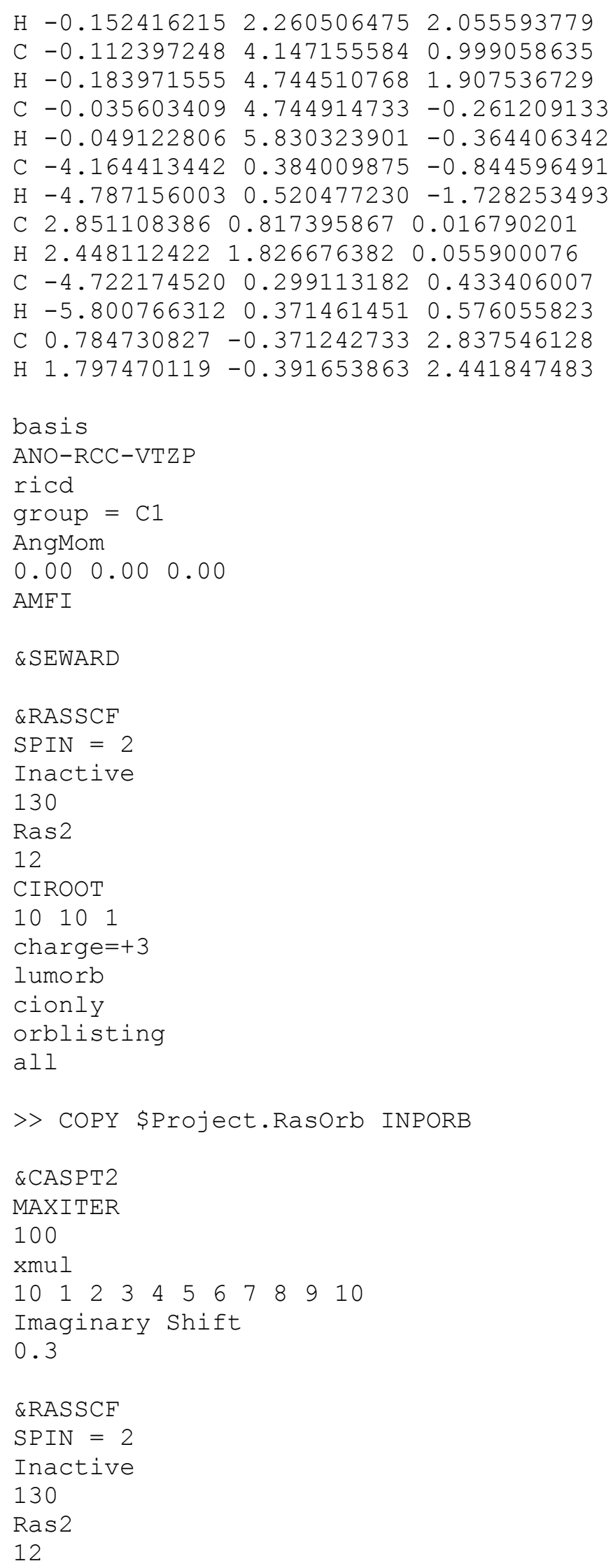




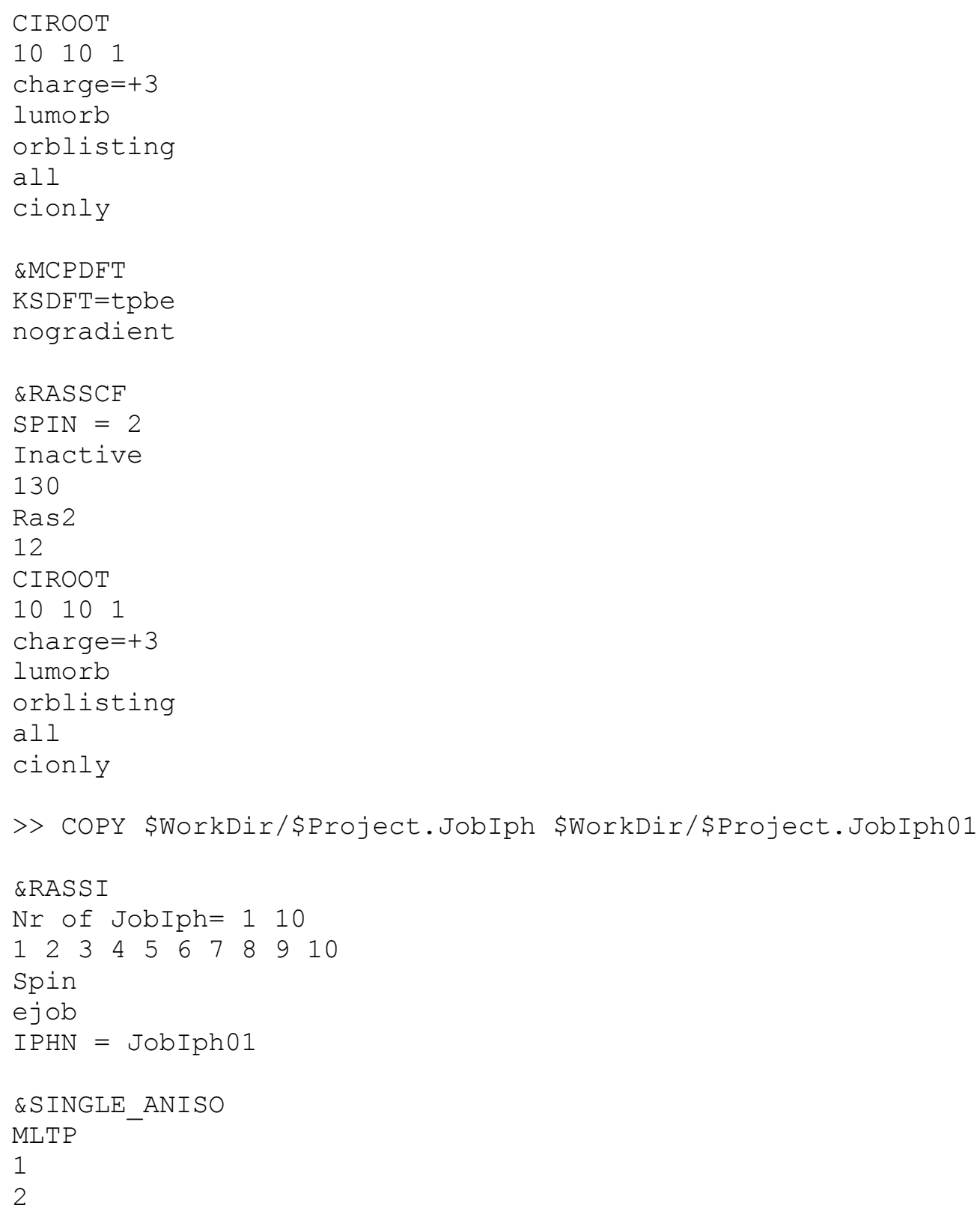




\section{Section S5}

Table S3.1. $g$ tensor values $(\Delta g)$ for $\mathrm{d}^{9}$ configurations by CASSCF, CASPT2, XMS-CASPT2, MCPDFT, and XMS-PDFT. An IPEA shift of 0.25 a.u. and imaginary shift of 0.3 a.u. are used for CASPT2 and XMS-CASPT2. ${ }^{a}$ For MC-PDFT and XMS-PDFT calculations, the tPBE functional is used.

\begin{tabular}{|c|c|c|c|c|c|c|c|}
\hline Molecule & & SA(5)-CASSCF & CASPT2 & XMS-CASPT2 & MC-PDFT & XMS-PDFT & Exp. \\
\hline \multirow{3}{*}[\mathrm{Cu}(\mathrm{NH}_{3})_{4}]{$^{2+}$} & $\Delta g_{\min }$ & 73 & 68 & 67 & 64 & 64 & 44 \\
\hline & $\Delta g_{\text {mid }}$ & 73 & 68 & 67 & 64 & 64 & 44 \\
\hline & $\Delta g_{\max }$ & 409 & 357 & 350 & 330 & 330 & 238 \\
\hline \multirow{3}{*}[\mathrm{Cu}(\text{py})_{4}]{$^{2+}$} & $\Delta g_{\min }$ & 66 & & & 58 & 58 & 50 \\
\hline & $\Delta g_{\text {mid }}$ & 66 & $b$ & $b$ & 58 & 58 & 50 \\
\hline & $\Delta g_{\max }$ & 367 & & & 300 & 300 & 260 \\
\hline \multirow{3}{*}[\mathrm{Cu}(\mathrm{iz})_{4}]{$^{2+}$} & $\Delta g_{\min }$ & 64 & 60 & 59 & 57 & 57 & 48 \\
\hline & $\Delta g_{\text {mid }}$ & 65 & 60 & 59 & 58 & 58 & 48 \\
\hline & $\Delta g_{\max }$ & 351 & 309 & 303 & 293 & 293 & 259 \\
\hline \multirow{3}{*}[\mathrm{Cu}(\mathrm{en})_{2}]{$^{2+}$} & $\Delta g_{\min }$ & 63 & 58 & 57 & 55 & 55 & 38 \\
\hline & $\Delta g_{\text {mid }}$ & 64 & 59 & 57 & 56 & 56 & 38 \\
\hline & $\Delta g_{\max }$ & 343 & 295 & 288 & 278 & 278 & 203 \\
\hline \multirow{3}{*}{$\mathrm{Cu}(\mathrm{gly})_{2}$} & $\Delta g_{\min }$ & 56 & 52 & 48 & 50 & 49 & 49 \\
\hline & $\Delta g_{\text {mid }}$ & 86 & 80 & 81 & 75 & 76 & 49 \\
\hline & $\Delta g_{\max }$ & 386 & 334 & 322 & 314 & 316 & 264 \\
\hline \multirow{3}{*}{$\mathrm{Cu}(\mathrm{ox})_{2}$} & $\Delta g_{\min }$ & 63 & 59 & 48 & 56 & 58 & 49 \\
\hline & $\Delta g_{\text {mid }}$ & 84 & 78 & 87 & 74 & 72 & 49 \\
\hline & $\Delta g_{\max }$ & 378 & 327 & 314 & 312 & 313 & 199 \\
\hline \multirow{3}{*}{$\mathrm{Cu}(\mathrm{sac})_{2}$} & $\Delta g_{\min }$ & 69 & 63 & 39 & 61 & 58 & 37 \\
\hline & $\Delta g_{\text {mid }}$ & 74 & 69 & 96 & 66 & 69 & 47 \\
\hline & $\Delta g_{\max }$ & 368 & 323 & 314 & 307 & 308 & 197 \\
\hline \multirow{3}{*}{$\mathrm{Cu}(\mathrm{acac})_{2}$} & $\Delta g_{\min }$ & 77 & 72 & 69 & 68 & 68 & 50 \\
\hline & $\Delta g_{\text {mid }}$ & 77 & 72 & 71 & 69 & 69 & 50 \\
\hline & $\Delta g_{\max }$ & 448 & 390 & 379 & 367 & 367 & 263 \\
\hline \multirow{3}{*}{$\mathrm{Cu}(\mathrm{kts})_{2}$} & $\Delta g_{\min }$ & 69 & 60 & 58 & 58 & 58 & 48 \\
\hline & $\Delta g_{\text {mid }}$ & 73 & 64 & 62 & 62 & 62 & 48 \\
\hline & $\Delta g_{\max }$ & 328 & 276 & 265 & 264 & 264 & 136 \\
\hline \multirow{3}{*}{$\mathrm{Cu}(\mathrm{dtc})_{2}$} & $\Delta g_{\min }$ & 75 & 63 & 59 & 60 & 60 & 15 \\
\hline & $\Delta g_{\text {mid }}$ & 76 & 64 & 60 & 61 & 61 & 20 \\
\hline & $\Delta g_{\max }$ & 352 & 280 & 261 & 266 & 266 & 79 \\
\hline \multirow{3}{*}[\mathrm{Ni}(\text{cyclam})]{$^{+}$} & $\Delta g_{\min }$ & 58 & 49 & 48 & 58 & 58 & 48 \\
\hline & $\Delta g_{\text {mid }}$ & 59 & 50 & 50 & 60 & 60 & 48 \\
\hline & $\Delta g_{\max }$ & 314 & 286 & 278 & 293 & 294 & 136 \\
\hline \multirow{3}{*}{$\mathrm{NiH}(\mathrm{CO})_{3}$} & $\Delta g_{\min }$ & -4 & -3 & -3 & -3 & -3 & -2 \\
\hline & $\Delta g_{\text {mid }}$ & 135 & 116 & 115 & 128 & 128 & 65 \\
\hline & $\Delta g_{\max }$ & 135 & 116 & 115 & 129 & 128 & 65 \\
\hline $\operatorname{MUE}(11)^{c}$ & & 72 & 52 & 49 & 48 & 47 & \\
\hline $\operatorname{MURE}(11)^{c}$ & & 0.50 & 0.37 & 0.34 & 0.35 & 0.35 & \\
\hline $\operatorname{MUE}(12)^{d}$ & & 70 & / & / & 44 & 44 & \\
\hline $\operatorname{MURE}(12)^{d}$ & & 0.47 & I & I & 0.33 & 0.33 & \\
\hline
\end{tabular}

${ }^{a}$ Note that in the article proper, we put the XMS-CASPT2 results without IPEA, which is recommended by Schapiro. But in the SI, we show only the XMS-CASPT2 results with the default value of 0.25 a.u. for the IPEA.

${ }^{b}$ No values because of too expensive computational cost.

${ }^{c}$ The errors are averaged over all the systems without $\left[\mathrm{Cu}(\mathrm{py})_{4}\right]^{2+}$.

${ }^{d}$ The errors are averaged over all the 12 systems. 
Table S3.2. $g$ tensor values $(\Delta g)$ for $\mathrm{d}^{7}$ configurations at CASSCF, CASPT2, XMS-CASPT2, MCPDFT and XMS-PDFT levels. An IPEA shift of 0.25 a.u. and an imaginary shift of 0.3 a.u. are used for CASPT2 and XMS-CASPT2. For MC-PDFT and XMS-PDFT calculations, tPBE functional is used.

\begin{tabular}{|c|c|c|c|c|c|c|c|}
\hline Molecule & & $\begin{array}{c}\text { SA(5)- } \\
\text { CASSCF }\end{array}$ & CASPT2 & $\begin{array}{c}\text { XMS- } \\
\text { CASPT2 }\end{array}$ & $\begin{array}{c}\text { MC- } \\
\text { PDFT }\end{array}$ & $\begin{array}{l}\text { XMS- } \\
\text { PDFT }\end{array}$ & Exp. \\
\hline \multirow{3}{*}{ Co(acacen) } & $\Delta g_{\min }$ & -1162 & -147 & -276 & -1430 & -1754 & -78 \\
\hline & $\Delta g_{\text {mid }}$ & -1127 & 127 & -24 & -1288 & -1665 & 8 \\
\hline & $\Delta g_{\max }$ & 3276 & 1733 & 2295 & 3417 & 3538 & 1187 \\
\hline \multirow{3}{*}{$\mathrm{Co}(\operatorname{sacsac})_{2}$} & $\Delta g_{\min }$ & -1297 & -586 & -822 & -551 & -583 & -103 \\
\hline & $\Delta g_{\text {mid }}$ & -1167 & -525 & -762 & -331 & -220 & -98 \\
\hline & $\Delta g_{\max }$ & 3435 & 2698 & 3397 & 2590 & 2089 & 1277 \\
\hline \multirow{3}{*}{ Co(tacacen $)$} & $\Delta g_{\min }$ & -1576 & -644 & -424 & -1620 & -1083 & -57 \\
\hline & $\Delta g_{\text {mid }}$ & -1537 & -572 & -297 & -1545 & -1064 & -22 \\
\hline & $\Delta g_{\max }$ & 3531 & 2832 & 2649 & 3543 & 2855 & 1044 \\
\hline \multirow{3}{*}[\mathrm{Ni}(\mathrm{mnt})_{2}]{$^{-}$} & $\Delta g_{\min }$ & -1394 & -198 & -380 & -1477 & -1215 & -4 \\
\hline & $\Delta g_{\text {mid }}$ & -1112 & 58 & -10 & -1266 & -613 & 39 \\
\hline & $\Delta g_{\max }$ & 3378 & 1943 & 2482 & 3420 & 1715 & 157 \\
\hline MUE & & 1668 & 666 & 820 & 1542 & 1201 & \\
\hline MURE & & 4.09 & 1.50 & 1.96 & 4.12 & 2.62 & \\
\hline
\end{tabular}


Table S3.3. $g$ tensor values $(\Delta g)$ for $\mathrm{d}^{5}$ configurations at CASSCF, MC-PDFT and XMS-PDFT levels. For MC-PDFT and XMS-PDFT calculations, tPBE functional is used.

\begin{tabular}{|c|c|c|c|c|c|c|}
\hline Molecules & & $\begin{array}{c}\text { SA(5)- } \\
\text { CASSCF }\end{array}$ & $\begin{array}{l}\text { MC- } \\
\text { PDFT }\end{array}$ & $\begin{array}{l}\text { XMS- } \\
\text { PDFT }\end{array}$ & $\mathrm{NEVPT}^{a}$ & Exp. \\
\hline \multirow{3}{*}[\mathrm{Fe}(\mathrm{bpy})_{3}]{$^{3+}$} & $\Delta g_{\min }$ & -430 & -273 & -491 & -402 & -392 \\
\hline & $\Delta g_{\text {mid }}$ & 789 & 653 & 726 & 778 & 688 \\
\hline & $\Delta g_{\max }$ & 830 & 726 & 935 & 783 & 688 \\
\hline \multirow{3}{*}[\mathrm{Fe}(\mathrm{N}_{3})(\mathrm{MePy}_{2}\mathrm{tacn})]{$^{2+}$} & $\Delta g_{\min }$ & -724 & -334 & -323 & -425 & -172 \\
\hline & $\Delta g_{\text {mid }}$ & 172 & 219 & 329 & 301 & 258 \\
\hline & $\Delta g_{\max }$ & 1581 & 1158 & 1039 & 1163 & 578 \\
\hline \multirow{3}{*}[\mathrm{Fe}(\mathrm{PyMS})_{2}]{$^{+}$} & $\Delta g_{\min }$ & -20 & -14 & -7 & -47 & 28 \\
\hline & $\Delta g_{\text {mid }}$ & 176 & 163 & 147 & 106 & 88 \\
\hline & $\Delta g_{\max }$ & 308 & 270 & 234 & 170 & 168 \\
\hline \multirow{3}{*}[\mathrm{Fe}(\mathrm{PyAS})_{2}]{$^{+}$} & $\Delta g_{\min }$ & -35 & -30 & -31 & -10 & 8 \\
\hline & $\Delta g_{\text {mid }}$ & 235 & 222 & 224 & 93 & 118 \\
\hline & $\Delta g_{\max }$ & 340 & 317 & 324 & 154 & 168 \\
\hline \multirow{3}{*}{$\begin{array}{l}\mathrm{Mn}(\mathrm{dmpe})_{2}(o- \\
\text { xylylene })\end{array}$} & $\Delta g_{\min }$ & -33 & -34 & -33 & -31 & -2 \\
\hline & $\Delta g_{\text {mid }}$ & 83 & 85 & 83 & 81 & 33 \\
\hline & $\Delta g_{\max }$ & 97 & 96 & 94 & 90 & 108 \\
\hline MUE & & 175 & 105 & 108 & 88 & \\
\hline MURE & & 0.51 & 0.37 & 0.36 & 0.24 & \\
\hline
\end{tabular}

${ }^{a}$ The NEVPT2 results are taken from Ref. 38. 
Table S3.4. $g$ tensor values $(\Delta g)$ for $\mathrm{d}^{1}$ configurations at CASSCF, CASPT2, XMS-CASPT2, MCPDFT and XMS-PDFT levels. An IPEA shift of 0.25 a.u. and an imaginary shift of 0.3 a.u. are used for CASPT2 and XMS-CASPT2. For MC-PDFT and XMS-PDFT calculations, tPBE functional is used.

\begin{tabular}{|c|c|c|c|c|c|c|c|}
\hline Molecule & & $\begin{array}{c}\text { SA(5)- } \\
\text { CASSCF } \\
\end{array}$ & CASPT2 & $\begin{array}{c}\text { XMS- } \\
\text { CASPT2 } \\
\end{array}$ & $\begin{array}{l}\text { MC- } \\
\text { PDFT } \\
\end{array}$ & $\begin{array}{l}\text { XMS- } \\
\text { PDFT } \\
\end{array}$ & Exp. \\
\hline \multirow{3}{*}[\mathrm{MnN}(\mathrm{CN})_{4}]{$^{-}$} & $\Delta g_{\min }$ & -45 & -40 & -38 & -41 & -37 & -3 \\
\hline & $\Delta g_{\text {mid }}$ & 7 & 7 & 5 & 8 & 5 & 2 \\
\hline & $\Delta g_{\max }$ & 7 & 7 & 5 & 8 & 5 & 2 \\
\hline \multirow{3}{*}{$\begin{array}{l}{[\mathrm{MnN}(\text { cyclam })} \\
\left.\left(\mathrm{NCCH}_{3}\right)\right]^{3+}\end{array}$} & $\Delta g_{\min }$ & -74 & -64 & -56 & -65 & -74 & -3 \\
\hline & $\Delta g_{\text {mid }}$ & 6 & 6 & 4 & 7 & 2 & -3 \\
\hline & $\Delta g_{\max }$ & 7 & 7 & 4 & 9 & 4 & 7 \\
\hline \multirow{3}{*}{$\mathrm{VO}(\mathrm{acac})_{2}$} & $\Delta g_{\min }$ & -87 & -77 & -72 & -76 & -76 & -57 \\
\hline & $\Delta g_{\text {mid }}$ & -24 & -19 & -15 & -19 & -19 & -21 \\
\hline & $\Delta g_{\max }$ & -21 & -16 & -13 & -17 & -17 & -21 \\
\hline \multirow{3}{*}[\mathrm{VO}(\mathrm{H}_{2}\mathrm{O})_{5}]{$^{2+}$} & $\Delta g_{\min }$ & -101 & -88 & -84 & -88 & -88 & -65 \\
\hline & $\Delta g_{\text {mid }}$ & -21 & -15 & -12 & -16 & -16 & -18 \\
\hline & $\Delta g_{\max }$ & -20 & -15 & -12 & -15 & -15 & -10 \\
\hline MUE & & 18 & 15 & 13 & 15 & 15 & \\
\hline MURE & & 2.51 & 2.22 & 1.97 & 2.34 & 2.13 & \\
\hline
\end{tabular}


Table S3.5. $g$ tensor values $(\Delta g)$ for $\mathrm{d}^{9}$ configurations at CASCI, MC-PDFT and XMS-PDFT levels with CASCI scheme. For MC-PDFT and XMS-PDFT calculations, tPBE functional is used.

\begin{tabular}{|c|c|c|c|c|c|}
\hline Molecule & & CASCI $(1,10)$ & $\mathrm{MC}(1,10)-\mathrm{PDFT}$ & XMS(1,10)-PDFT & Exp. \\
\hline \multirow{3}{*}[\mathrm{Cu}(\mathrm{NH}_{3})_{4}]{$^{2+}$} & $\Delta g_{\min }$ & 65 & 57 & 51 & 44 \\
\hline & $\Delta g_{\text {mid }}$ & 65 & 58 & 51 & 44 \\
\hline & $\Delta g_{\max }$ & 372 & 312 & 280 & 238 \\
\hline \multirow{3}{*}[\mathrm{Cu}(\mathrm{py})_{4}]{$^{2+}$} & $\Delta g_{\min }$ & 59 & 52 & 39 & 50 \\
\hline & $\Delta g_{\text {mid }}$ & 59 & 52 & 39 & 50 \\
\hline & $\Delta g_{\max }$ & 332 & 280 & 189 & 260 \\
\hline \multirow{3}{*}[\mathrm{Cu}(\mathrm{iz})_{4}]{$^{2+}$} & $\Delta g_{\min }$ & 58 & 52 & 40 & 48 \\
\hline & $\Delta g_{\text {mid }}$ & 58 & 52 & 40 & 48 \\
\hline & $\Delta g_{\max }$ & 320 & 276 & 192 & 259 \\
\hline \multirow{3}{*}[\mathrm{Cu}(\mathrm{en})_{2}]{$^{2+}$} & $\Delta g_{\min }$ & 56 & 49 & 38 & 38 \\
\hline & $\Delta g_{\text {mid }}$ & 57 & 50 & 38 & 38 \\
\hline & $\Delta g_{\max }$ & 308 & 258 & 179 & 203 \\
\hline \multirow{3}{*}{$\mathrm{Cu}(\text { gly })_{2}$} & $\Delta g_{\min }$ & 51 & 45 & 33 & 49 \\
\hline & $\Delta g_{\text {mid }}$ & 76 & 66 & 41 & 49 \\
\hline & $\Delta g_{\max }$ & 349 & 294 & 283 & 264 \\
\hline \multirow{3}{*}{$\mathrm{Cu}(\mathrm{ox})_{2}$} & $\Delta g_{\min }$ & 56 & 50 & 41 & 49 \\
\hline & $\Delta g_{\text {mid }}$ & 74 & 65 & 60 & 49 \\
\hline & $\Delta g_{\max }$ & 345 & 295 & 296 & 199 \\
\hline \multirow{3}{*}{$\mathrm{Cu}(\mathrm{sac})_{2}$} & $\Delta g_{\min }$ & 60 & 53 & 40 & 37 \\
\hline & $\Delta g_{\text {mid }}$ & 66 & 58 & 43 & 47 \\
\hline & $\Delta g_{\max }$ & 333 & 287 & 197 & 197 \\
\hline \multirow{3}{*}{$\mathrm{Cu}(\mathrm{acac})_{2}$} & $\Delta g_{\min }$ & 69 & 61 & 48 & 50 \\
\hline & $\Delta g_{\text {mid }}$ & 69 & 61 & 49 & 50 \\
\hline & $\Delta g_{\max }$ & 408 & 347 & 261 & 263 \\
\hline \multirow{3}{*}{$\mathrm{Cu}(\mathrm{kts})_{2}$} & $\Delta g_{\min }$ & 57 & 49 & 35 & 48 \\
\hline & $\Delta g_{\text {mid }}$ & 60 & 50 & 35 & 48 \\
\hline & $\Delta g_{\max }$ & 277 & 227 & 153 & 136 \\
\hline \multirow{3}{*}{$\mathrm{Cu}(\mathrm{dtc})_{2}$} & $\Delta g_{\min }$ & 54 & 43 & 36 & 15 \\
\hline & $\Delta g_{\text {mid }}$ & 55 & 43 & 36 & 20 \\
\hline & $\Delta g_{\max }$ & 260 & 198 & 161 & 79 \\
\hline \multirow{3}{*}[\mathrm{Ni}(\text{cyclam})]{$^{+}$} & $\Delta g_{\min }$ & 48 & 48 & 47 & 48 \\
\hline & $\Delta g_{\text {mid }}$ & 49 & 49 & 48 & 48 \\
\hline & $\Delta g_{\max }$ & 254 & 250 & 245 & 136 \\
\hline \multirow{3}{*}{$\mathrm{NiH}(\mathrm{CO})_{3}$} & $\Delta g_{\min }$ & -2 & -2 & -2 & -2 \\
\hline & $\Delta g_{\text {mid }}$ & 91 & 88 & 89 & 65 \\
\hline & $\Delta g_{\max }$ & 91 & 88 & 89 & 65 \\
\hline MUE & & 48 & 29 & 21 & \\
\hline MURE & & 0.31 & 0.20 & 0.15 & \\
\hline
\end{tabular}


Table S3.6. $g$ tensor values $(\Delta g)$ for $\mathrm{d}^{7}$ configurations at CASCI, MC-PDFT and XMS-PDFT levels with CASCI scheme. For MC-PDFT and XMS-PDFT calculations, tPBE functional is used.

\begin{tabular}{cccccc}
\hline Molecules & & CASCI(1,10) & MC(1,10)-PDFT & XMS(1,10)-PDFT & Exp. \\
\hline \multirow{2}{*}{ Co(acacen) $)$} & $\Delta g_{\text {min }}$ & -187 & -183 & -174 & -78 \\
& $\Delta g_{\text {mid }}$ & 363 & 250 & 250 & 8 \\
& $\Delta g_{\max }$ & 1685 & 1660 & 1627 & 1187 \\
& $\Delta g_{\min }$ & -182 & -138 & -190 & -103 \\
Co(sacsac) 2 & $\Delta g_{\operatorname{mid}}$ & 126 & 116 & 62 & -98 \\
& $\Delta g_{\max }$ & 1735 & 1537 & 1731 & 1277 \\
& $\Delta g_{\min }$ & -168 & -188 & -184 & -57 \\
Co(tacacen) & $\Delta g_{\operatorname{mid}}$ & 200 & 104 & 106 & -22 \\
& $\Delta g_{\max }$ & 1606 & 1670 & 1654 & 1044 \\
& $\Delta g_{\min }$ & -116 & -82 & -101 & -4 \\
$\left.[\text { Ni(mnt })_{2}\right]^{-}$ & $\Delta g_{\operatorname{mid}}$ & 688 & 632 & 607 & 39 \\
& $\Delta g_{\max }$ & 1408 & 1266 & 1392 & 157 \\
MUE & & 386 & 333 & 354 & \\
MURE & & 1.26 & 1.11 & 1.18 & \\
\hline
\end{tabular}


Table S3.7. $g$ tensor values $(\Delta g)$ for $\mathrm{d}^{5}$ configurations at CASCI, MC-PDFT and XMS-PDFT levels with CASCI scheme. For MC-PDFT and XMS-PDFT calculations, tPBE functional is used.

\begin{tabular}{|c|c|c|c|c|c|c|}
\hline Molecules & & $\begin{array}{c}\operatorname{CASCI}(N, 10 \\
)\end{array}$ & $\begin{array}{c}\mathrm{MC}(N, 10) \\
-\mathrm{PDFT} \\
\end{array}$ & $\begin{array}{c}\mathrm{XMS}(N, 10) \\
\text {-PDFT }\end{array}$ & NEVPT2 $^{a}$ & Exp. \\
\hline \multirow{3}{*}[\mathrm{Fe}(\mathrm{bpy})_{3}]{$^{3+}$} & $\Delta g_{\min }$ & $-62^{b}$ & $-73^{b}$ & $-71^{b}$ & -402 & -392 \\
\hline & $\Delta g_{\text {mid }}$ & $314^{b}$ & $337^{b}$ & $335^{b}$ & 778 & 688 \\
\hline & $\Delta g_{\max }$ & $315^{b}$ & $341^{b}$ & $337^{b}$ & 783 & 688 \\
\hline \multirow{4}{*}[\mathrm{Fe}(\mathrm{N}_{3})(\mathrm{MePy}_{2}\operatorname{tacn})]{$^{2+}$} & $\Delta g_{\min }$ & -110 & -95 & -94 & -425 & -172 \\
\hline & $\Delta g_{\text {mid }}$ & 239 & 230 & 235 & 301 & 258 \\
\hline & $\Delta g_{\max }$ & 469 & 409 & 405 & 1163 & 578 \\
\hline & $\Delta g_{\min }$ & -14 & -9 & -14 & -47 & 28 \\
\hline \multirow[t]{3}{*}[\mathrm{Fe}(\mathrm{PyMS})_{2}]{$^{+}$} & $\Delta g_{\text {mid }}$ & 106 & 95 & 95 & 106 & 88 \\
\hline & $\Delta g_{\max }$ & 199 & 178 & 224 & 170 & 168 \\
\hline & $\Delta g_{\min }$ & -26 & -23 & -46 & -10 & 8 \\
\hline \multirow[t]{2}{*}[\mathrm{Fe}(\mathrm{PyAS})_{2}]{$^{+}$} & $\Delta g_{\text {mid }}$ & 142 & 133 & 123 & 93 & 118 \\
\hline & $\Delta g_{\max }$ & 204 & 191 & 357 & 154 & 168 \\
\hline \multirow{3}{*}{$\begin{array}{l}\mathrm{Mn}(\text { dmpe })_{2}(o- \\
\quad \text { xylylene })\end{array}$} & $\Delta g_{\min }$ & -13 & -16 & -11 & -31 & -2 \\
\hline & $\Delta g_{\text {mid }}$ & 56 & 56 & 56 & 81 & 33 \\
\hline & $\Delta g_{\max }$ & 58 & 57 & 57 & 90 & 108 \\
\hline MUE & & 102 & 100 & 116 & 88 & \\
\hline MURE & & 0.25 & 0.23 & 0.32 & 0.24 & \\
\hline
\end{tabular}

${ }^{a}$ The NEVPT2 results are taken from Ref. 38 .

${ }^{b}$ The CASCI calculation is based on orbitals optimized for the first three low-lying states. 
Table S3.8. $g$ tensor values $(\Delta g)$ for $\mathrm{d}^{1}$ configurations at CASCI, MC-PDFT and XMS-PDFT levels with CASCI scheme. For MC-PDFT and XMS-PDFT calculations, tPBE functional is used.

\begin{tabular}{|c|c|c|c|c|c|}
\hline Molecules & & $\operatorname{CASCI}(1, R)$ & $\begin{array}{l}\mathrm{MC}(1, R)- \\
\mathrm{PDFT} \\
\end{array}$ & $\begin{array}{c}\text { XMS }(1, R)- \\
\text { PDFT }\end{array}$ & Exp. \\
\hline \multirow{6}{*}[\mathrm{MnN}(\text{cyclam})(\mathrm{NCCH}_{3})]{$^{3+}$} & $\Delta g_{\min }$ & 0 & 0 & 0 & -3 \\
\hline & $\Delta g_{\text {mid }}$ & 3 & 4 & 5 & 2 \\
\hline & $\Delta g_{\max }$ & 3 & 4 & 5 & 2 \\
\hline & $\Delta g_{\min }$ & 0 & 0 & 0 & -3 \\
\hline & $\Delta g_{\text {mid }}$ & 4 & 5 & 6 & -3 \\
\hline & $\Delta g_{\max }$ & 5 & 5 & 6 & 7 \\
\hline \multirow{3}{*}{$\mathrm{VO}(\mathrm{acac})_{2}$} & $\Delta g_{\min }$ & -83 & -78 & -78 & -57 \\
\hline & $\Delta g_{\text {mid }}$ & -21 & -18 & -18 & -21 \\
\hline & $\Delta g_{\max }$ & -18 & -15 & -15 & -21 \\
\hline \multirow{3}{*}[\mathrm{VO}(\mathrm{H}_{2}\mathrm{O})_{5}]{$^{2+}$} & $\Delta g_{\min }$ & -96 & -92 & -91 & -65 \\
\hline & $\Delta g_{\text {mid }}$ & -19 & -15 & -15 & -18 \\
\hline & $\Delta g_{\max }$ & -18 & -14 & -14 & -10 \\
\hline MUE & & 7 & 7 & 7 & \\
\hline MURE & & 0.38 & 0.44 & 0.49 & \\
\hline
\end{tabular}




\section{Section 6. A note on the CAS and RAS nomenclature used in the article}

Although the nomenclature is explained in the article as the methods are introduced, we summarize it here all in one place to be sure there is no mistaking our meaning.

Our CAS calculations ae labeled as either CASSCF or CASCI. In the CASSCF scheme, five roots are averaged [i.e., we use $\mathrm{SA}(5)-\mathrm{CASSCF}$ ] for orbital optimization and are used for the following magnetic calculation. In the scheme labeled as CASCI, the orbitals are optimized for only the ground state, and then CASCI wave functions based on these orbitals are used for the following magnetic calculation.

In contrast, all RAS calculations in this article are properly labeled as RASCI (not RASSCF) because we did not optimize the orbitals for the RAS space, but instead used orbitals optimized by CASSCF with a smaller active space. In section 4.1 .2 of the article, the RASCI calculations are based on orbitals optimized by SA(5)-CASSCF, whereas in section 4.3, the RASCI calculations are based on orbitals optimized by state-specific CASSCF. Consequently, using the notation defined in Section 3, we label the calculations in Section 4.1.2 as $\operatorname{RASCI}(5,5)$ and the calculations in Section 4.3 as RASCI $(1,10)$. 


\section{Section $\mathbf{S 7}$}

Table S4. $g$ tensor values $(\Delta g)$ for the $\left[\mathrm{Ni}(\mathrm{mnt})_{2}\right]^{-}$molecule with RAS calculations. Five $3 \mathrm{~d}$ orbitals of the $\mathrm{Ni}$ atom are included in the RAS2 space, and various numbers of orbitals are involved in RAS1 and RAS3 spaces. For PDFT calculations, the tPBE-HLE functional is used.

\begin{tabular}{|c|c|c|c|c|c|c|}
\hline RAS1 & RAS3 & & $\operatorname{RASCI}(5,5)$ & RASCI(5,5)-PDFT & RASCI $(1,10)$ & RASCI $(1,10)-\mathrm{PDFT}$ \\
\hline \multirow{3}{*}{5} & \multirow{3}{*}{5} & $\Delta g_{\min }$ & -1099 & -293 & -114 & -37 \\
\hline & & $\Delta g_{\text {mid }}$ & -681 & 375 & 717 & 605 \\
\hline & & $\Delta g_{\max }$ & 3202 & 1984 & 1400 & 956 \\
\hline \multirow{3}{*}{5} & \multirow{3}{*}{30} & $\Delta g_{\min }$ & -1164 & -293 & -117 & -37 \\
\hline & & $\Delta g_{\text {mid }}$ & -777 & 370 & 712 & 601 \\
\hline & & $\Delta g_{\max }$ & 3248 & 1981 & 1418 & 955 \\
\hline \multirow{3}{*}{13} & \multirow{3}{*}{5} & $\Delta g_{\min }$ & -1579 & -828 & -323 & -240 \\
\hline & & $\Delta g_{\text {mid }}$ & -1528 & -414 & 394 & 331 \\
\hline & & $\Delta g_{\max }$ & 3361 & 2880 & 2093 & 1884 \\
\hline \multirow{3}{*}{13} & \multirow{3}{*}{10} & $\Delta g_{\min }$ & -1475 & -988 & -345 & -274 \\
\hline & & $\Delta g_{\text {mid }}$ & -1431 & -644 & 361 & 270 \\
\hline & & $\Delta g_{\max }$ & 3328 & 3036 & 2135 & 1967 \\
\hline \multirow{3}{*}{13} & \multirow{3}{*}{20} & $\Delta g_{\min }$ & -1510 & -1088 & -341 & -289 \\
\hline & & $\Delta g_{\text {mid }}$ & -1463 & -781 & 370 & 243 \\
\hline & & $\Delta g_{\max }$ & 3339 & 3119 & 2125 & 2007 \\
\hline \multirow{3}{*}{13} & \multirow{3}{*}{30} & $\Delta g_{\min }$ & -1482 & -1205 & -347 & -318 \\
\hline & & $\Delta g_{\text {mid }}$ & -1437 & -932 & 361 & 203 \\
\hline & & $\Delta g_{\max }$ & 3329 & 3202 & 2140 & 2079 \\
\hline \multirow{3}{*}{20} & \multirow{3}{*}{20} & $\Delta g_{\min }$ & -1956 & -715 & -276 & -242 \\
\hline & & $\Delta g_{\text {mid }}$ & -1941 & -300 & 446 & 291 \\
\hline & & $\Delta g_{\max }$ & 3417 & 2719 & 1940 & 1865 \\
\hline \multirow{3}{*}{25} & \multirow{3}{*}{20} & $\Delta g_{\min }$ & -1988 & -760 & -291 & -277 \\
\hline & & $\Delta g_{\text {mid }}$ & -1892 & -364 & 415 & 236 \\
\hline & & $\Delta g_{\max }$ & 3410 & 2774 & 1982 & 1958 \\
\hline
\end{tabular}

This table shows the results of RASCI calculations carried out to study the effect of coreorbital spin polarization. In the RASCI calculations, the orbitals are optimized at the CASSCF $(9 \mathrm{e}, 11 \mathrm{o})$ level, and this is followed by a RASCI calculation, i.e., a configuration interaction calculation in a larger, but incomplete active space and without orbital re-optimization. The five $3 \mathrm{~d}$ orbitals of Ni atom are included in the RAS2 space; the in-plane $\sigma$ bonding orbital, 3s and 3p orbitals of Ni atom and the several doubly occupied orbitals with the highest orbital energies are included in the RAS1 space; the five $3 \mathrm{~d}^{\prime}$ orbitals and several virtual orbitals with the lowest orbital energies are included in the RAS3 space; and at most one hole and one electron are allowed respectively in the RAS1 and RAS3 spaces. (We capped the number of orbitals in the RAS3 space at 30 because our results show the number of orbitals in RAS3 doesn't have much effect on the results.) Overall, the RAS scheme employed in the calculation with core-orbital spin polarization does not improve the results in either wave function or PDFT calculations.

We also tried to involve 6 (one $\sigma+$ five $3 d$ ) and 11 (one $\sigma+$ five $3 d+$ five $3 d^{\prime}$ ) orbitals in the RAS2 space, and the corresponding results are quite close to those obtained with only five $3 \mathrm{~d}$ orbitals involved in the RAS2 space. 
Table S5.1. Absolute energies (in hartree) for $\mathrm{d}^{9}$ configurations at CASSCF, CASPT2, XMSCASPT2, MC-PDFT and XMS-PDFT levels. An IPEA shift of 0.25 a.u. and an imaginary shift of 0.3 a.u. are used for CASPT2 and XMS-CASPT2.

\begin{tabular}{|c|c|c|c|c|}
\hline Molecule & Spin-orbit state & SA(5)-CASSCF & CASPT2 & XMS-CASPT2 \\
\hline \multirow{9}{*}[\mathrm{Cu}(\mathrm{NH}_{3})_{4}]{$^{2+}$} & 1 & -1877.915600 & -1879.380595 & -1879.393551 \\
\hline & 2 & -1877.915600 & -1879.380595 & -1879.393551 \\
\hline & 3 & -1877.857125 & -1879.313200 & -1879.320022 \\
\hline & 4 & -1877.857125 & -1879.313200 & -1879.320022 \\
\hline & 5 & -1877.847338 & -1879.304479 & -1879.310510 \\
\hline & 6 & -1877.847338 & -1879.304479 & -1879.310510 \\
\hline & 7 & -1877.845254 & -1879.302149 & -1879.308694 \\
\hline & 8 & -1877.845254 & -1879.302149 & -1879.308694 \\
\hline & 9 & -1877.838236 & -1879.295332 & -1879.301447 \\
\hline \multirow{12}{*}[\mathrm{Cu}(\text{py})_{4}]{$^{2+}$} & 10 & -1877.838236 & -1879.295332 & -1879.301447 \\
\hline & 1 & -2640.544558 & \multirow{10}{*}{ (x) } & \multirow{10}{*}{$a$} \\
\hline & 2 & -2640.544558 & & \\
\hline & 3 & -2640.479636 & & \\
\hline & 4 & -2640.479636 & & \\
\hline & 5 & -2640.467030 & & \\
\hline & 6 & -2640.467030 & & \\
\hline & 7 & -2640.465208 & & \\
\hline & 8 & -2640.465208 & & \\
\hline & 9 & -2640.457975 & & \\
\hline & 10 & -2640.457975 & & \\
\hline & 1 & -2553.053842 & -2557.163885 & -2558.470414 \\
\hline \multirow{8}{*}[\mathrm{Cu}(\mathrm{iz})4]{$^{2+}$} & 2 & -2553.053842 & -2557.163885 & -2558.470414 \\
\hline & 3 & -2552.985999 & -2557.086573 & -2558.388786 \\
\hline & 4 & -2552.985999 & -2557.086573 & -2558.388786 \\
\hline & 5 & -2552.974157 & -2557.075822 & -2558.377757 \\
\hline & 6 & -2552.974157 & -2557.075822 & -2558.377757 \\
\hline & 7 & -2552.972664 & -2557.074162 & -2558.376179 \\
\hline & 8 & -2552.972664 & -2557.074162 & -2558.376179 \\
\hline & 9 & -2552.965114 & -2557.066767 & -2558.368709 \\
\hline \multirow{12}{*}[\mathrm{Cu}(\mathrm{en})_{2}]{$^{2+}$} & 10 & -2552.965114 & -2557.066767 & -2558.368709 \\
\hline & 1 & -2031.829454 & -2033.941277 & -2033.956079 \\
\hline & 2 & -2031.829454 & -2033.941277 & -2033.956079 \\
\hline & 3 & -2031.761588 & -2033.862195 & -2033.869413 \\
\hline & 4 & -2031.761588 & -2033.862195 & -2033.869413 \\
\hline & 5 & -2031.750827 & -2033.852635 & -2033.858918 \\
\hline & 6 & -2031.750827 & -2033.852635 & -2033.858918 \\
\hline & 7 & -2031.748883 & -2033.850450 & -2033.857178 \\
\hline & 8 & -2031.748883 & -2033.850450 & -2033.857178 \\
\hline & 9 & -2031.741690 & -2033.843449 & -2033.849709 \\
\hline & 10 & -2031.741690 & -2033.843449 & -2033.849709 \\
\hline & 1 & -2218.496954 & -2221.070247 & -2221.085107 \\
\hline \multirow{8}{*}{$\mathrm{Cu}(\text { gly })_{2}$} & 2 & -2218.496954 & -2221.070247 & -2221.085107 \\
\hline & 3 & -2218.434992 & -2220.998498 & -2221.005724 \\
\hline & 4 & -2218.434992 & -2220.998498 & -2221.005724 \\
\hline & 5 & -2218.426861 & -2220.991897 & -2220.999062 \\
\hline & 6 & -2218.426861 & -2220.991897 & -2220.999062 \\
\hline & 7 & -2218.419739 & -2220.983859 & -2220.989879 \\
\hline & 8 & -2218.419739 & -2220.983859 & -2220.989879 \\
\hline & 9 & -2218.412639 & -2220.976767 & -2220.982845 \\
\hline
\end{tabular}




\begin{tabular}{|c|c|c|c|c|}
\hline \multirow{10}{*}{$\mathrm{Cu}(\mathrm{ox})_{2}$} & 10 & -2218.412639 & -2220.976767 & -2220.982845 \\
\hline & 1 & -2601.469656 & -2605.724550 & -2605.740126 \\
\hline & 2 & -2601.469656 & -2605.724550 & -2605.740126 \\
\hline & 3 & -2601.406269 & -2605.651314 & -2605.658733 \\
\hline & 4 & -2601.406269 & -2605.651314 & -2605.658733 \\
\hline & 5 & -2601.399782 & -2605.646425 & -2605.653727 \\
\hline & 6 & -2601.399782 & -2605.646425 & -2605.653727 \\
\hline & 7 & -2601.394786 & -2605.640678 & -2605.647169 \\
\hline & 8 & -2601.394786 & -2605.640678 & -2605.647169 \\
\hline & 9 & -2601.387650 & -2605.633672 & -2605.639986 \\
\hline \multirow{11}{*}{$\mathrm{Cu}(\mathrm{sac})_{2}$} & 10 & -2601.387650 & -2605.633672 & -2605.639986 \\
\hline & 1 & -2449.869510 & -2453.503631 & -2453.517847 \\
\hline & 2 & -2449.869510 & -2453.503631 & -2453.517847 \\
\hline & 3 & -2449.804264 & -2453.429135 & -2453.436548 \\
\hline & 4 & -2449.804264 & -2453.429135 & -2453.436548 \\
\hline & 5 & -2449.796619 & -2453.423108 & -2453.430259 \\
\hline & 6 & -2449.796619 & -2453.423108 & -2453.430259 \\
\hline & 7 & -2449.793334 & -2453.419014 & -2453.425729 \\
\hline & 8 & -2449.793334 & -2453.419014 & -2453.425729 \\
\hline & 9 & -2449.785717 & -2453.411645 & -2453.417965 \\
\hline & 10 & -2449.785717 & -2453.411645 & -2453.417965 \\
\hline \multirow{10}{*}{$\mathrm{Cu}(\mathrm{acac})_{2}$} & 1 & -2340.366883 & -2343.491373 & -2343.505880 \\
\hline & 2 & -2340.366883 & -2343.491373 & -2343.505880 \\
\hline & 3 & -2340.311496 & -2343.427375 & -2343.434752 \\
\hline & 4 & -2340.311496 & -2343.427375 & -2343.434752 \\
\hline & 5 & -2340.299519 & -2343.416423 & -2343.423111 \\
\hline & 6 & -2340.299519 & -2343.416423 & -2343.423111 \\
\hline & 7 & -2340.298575 & -2343.414849 & -2343.421367 \\
\hline & 8 & -2340.298575 & -2343.414849 & -2343.421367 \\
\hline & 9 & -2340.289917 & -2343.406349 & -2343.412432 \\
\hline & 10 & -2340.289917 & -2343.406349 & -2343.412432 \\
\hline \multirow{10}{*}{$\mathrm{Cu}(\mathrm{kts})_{2}$} & 1 & -2932.701748 & -2935.466929 & -2935.485538 \\
\hline & 2 & -2932.701748 & -2935.466929 & -2935.485538 \\
\hline & 3 & -2932.636323 & -2935.388878 & -2935.397027 \\
\hline & 4 & -2932.636323 & -2935.388878 & -2935.397027 \\
\hline & 5 & -2932.632573 & -2935.385498 & -2935.393249 \\
\hline & 6 & -2932.632573 & -2935.385498 & -2935.393249 \\
\hline & 7 & -2932.629921 & -2935.382664 & -2935.390341 \\
\hline & 8 & -2932.629921 & -2935.382664 & -2935.390341 \\
\hline & 9 & -2932.623118 & -2935.376072 & -2935.383252 \\
\hline & 10 & -2932.623118 & -2935.376072 & -2935.383252 \\
\hline \multirow{10}{*}{$\mathrm{Cu}(\mathrm{dtc})_{2}$} & 1 & -3747.591001 & -3750.994355 & -3751.016016 \\
\hline & 2 & -3747.591001 & -3750.994355 & -3751.016016 \\
\hline & 3 & -3747.532725 & -3750.920518 & -3750.928538 \\
\hline & 4 & -3747.532725 & -3750.920518 & -3750.928538 \\
\hline & 5 & -3747.530062 & -3750.918116 & -3750.925791 \\
\hline & 6 & -3747.530062 & -3750.918116 & -3750.925791 \\
\hline & 7 & -3747.527043 & -3750.914506 & -3750.922457 \\
\hline & 8 & -3747.527043 & -3750.914506 & -3750.922457 \\
\hline & 9 & -3747.520945 & -3750.908891 & -3750.916139 \\
\hline & 10 & -3747.520945 & -3750.908891 & -3750.916139 \\
\hline \multirow{2}{*}{$\mathrm{Ni}($ cyclam $)]^{+}$} & 1 & -2130.043081 & -2133.146279 & -2134.245142 \\
\hline & 2 & -2130.043081 & -2133.146279 & -2134.245142 \\
\hline
\end{tabular}




\begin{tabular}{ccccc}
3 & -2129.979687 & -2133.076039 & -2134.177450 \\
4 & -2129.979687 & -2133.076039 & -2134.177450 \\
5 & -2129.965538 & -2133.054156 & -2134.166853 \\
6 & -2129.965538 & -2133.054156 & -2134.166853 \\
7 & -2129.963106 & -2133.051607 & -2134.164267 \\
8 & -2129.963106 & -2133.051607 & -2134.164267 \\
9 & -2129.953445 & -2133.040447 & -2134.152932 \\
& 10 & -2129.953445 & -2133.040447 & -2134.152932 \\
& 1 & -1858.350532 & -1860.059409 & -1860.076546 \\
$\mathrm{NiH}(\mathrm{CO})_{3}$ & 2 & -1858.350532 & -1860.059409 & -1860.076546 \\
& 3 & -1858.295290 & -1859.995435 & -1860.006356 \\
& 4 & -1858.295290 & -1859.995435 & -1860.006356 \\
& 5 & -1858.293186 & -1859.992891 & -1860.003143 \\
6 & -1858.293186 & -1859.992891 & -1860.003143 \\
& 7 & -1858.286283 & -1859.984593 & -1859.995769 \\
& 8 & -1858.286283 & -1859.984593 & -1859.995769 \\
9 & -1858.286251 & -1859.984200 & -1859.994716 \\
& 10 & -1858.286251 & -1859.984200 & -1859.994716 \\
\hline
\end{tabular}

${ }^{a}$ No values because of too expensive computational cost.

\begin{tabular}{cccccc}
\hline \multirow{2}{*}{ Molecule } & Spin-orbit state & MC-PDFT (tPBE) & XMS-PDFT (tPBE) & $\begin{array}{c}\text { MC-PDFT } \\
\text { (tPBE-HLE) }\end{array}$ & $\begin{array}{c}\text { XMS-PDFT } \\
\text { (tPBE-HLE) }\end{array}$ \\
\hline & 1 & -1880.082191 & -1880.082197 & -1902.928863 & -1902.928872 \\
{$\left[\mathrm{Cu}\left(\mathrm{NH}_{3}\right)_{4}\right]^{2+}$} & 2 & -1880.082191 & -1880.082197 & -1902.928863 & -1902.928872 \\
& 3 & -1880.009077 & -1880.009071 & -1902.865960 & -1902.865961 \\
& 4 & -1880.009077 & -1880.009071 & -1902.865960 & -1902.865961 \\
& 5 & -1880.000148 & -1880.000147 & -1902.858345 & -1902.858341 \\
& 7 & -1880.000148 & -1880.000147 & -1902.858345 & -1902.858341 \\
& 8 & -1879.997811 & -1879.997811 & -1902.854820 & -1902.854820 \\
{$\left[\mathrm{Cu}(\mathrm{py})_{4}\right]^{2+}$} & 9 & -1879.997811 & -1879.997811 & -1902.854820 & -1902.854820 \\
& 10 & -1879.990993 & -1879.990993 & -1902.848735 & -1902.848733 \\
& 1 & -1879.990993 & -1879.990993 & -1902.848735 & -1902.848733 \\
& 2 & -2646.512083 & -2646.512083 & -2693.772837 & -2693.772838 \\
& 3 & -2646.512083 & -2646.512083 & -2693.772837 & -2693.772838 \\
& 4 & -2646.432152 & -2646.432152 & -2693.705276 & -2693.705275 \\
& 5 & -2646.432152 & -2646.432152 & -2693.705276 & -2693.705275 \\
& 6 & -2646.420369 & -2646.420367 & -2693.695072 & -2693.695070 \\
& 7 & -2646.420369 & -2646.420367 & -2693.695072 & -2693.695070 \\
{$\left[\mathrm{Cu}(\mathrm{iz})_{4}\right]^{2+}$} & 8 & -2646.418373 & -2646.418371 & -2693.691746 & -2693.691743 \\
& 9 & -2646.418373 & -2646.418371 & -2693.691746 & -2693.691743 \\
& 10 & -2646.411284 & -2646.411282 & -2693.685507 & -2693.685504 \\
& 1 & -2646.411284 & -2646.411282 & -2693.685507 & -2693.685504 \\
& 2 & -2558.470416 & -2558.470414 & -2602.029597 & -2602.029596 \\
& 3 & -2558.470416 & -2558.470414 & -2602.029597 & -2602.029596 \\
& 4 & -2558.388781 & -2558.388786 & -2601.960942 & -2601.960948 \\
& 5 & -2558.388781 & -2558.388786 & -2601.960942 & -2601.960948 \\
& 7 & -2558.377761 & -2558.377757 & -2601.951450 & -2601.951442 \\
& 9 & -2558.377761 & -2558.377757 & -2601.951450 & -2601.951442 \\
& 9 & -2558.376181 & -2558.376179 & -2601.948517 & -2601.948519 \\
& 10 & -2558.376181 & -2558.376179 & -2601.948517 & -2601.948519 \\
& & -2558.368714 & -2558.368709 & -2601.942089 & -2601.942085 \\
& -2558.368714 & -2558.368709 & -2601.942089 & -2601.942085 \\
& & & & &
\end{tabular}




\begin{tabular}{|c|c|c|c|c|c|}
\hline & 1 & -2034.788162 & -2034.788162 & -2062.701687 & -2062.701687 \\
\hline & 2 & -2034.788162 & -2034.788162 & -2062.701687 & -2062.701687 \\
\hline & 3 & -2034.703893 & -2034.703929 & -2062.628688 & -2062.628772 \\
\hline & 4 & -2034.703893 & -2034.703929 & -2062.628688 & -2062.628772 \\
\hline \multirow{10}{*}[\mathrm{Cu}(\mathrm{en})_{2}]{$^{2+}$} & 5 & -2034.694055 & -2034.694057 & -2062.620261 & -2062.620197 \\
\hline & 6 & -2034.694055 & -2034.694057 & -2062.620261 & -2062.620197 \\
\hline & 7 & -2034.691988 & -2034.691985 & -2062.617031 & -2062.617078 \\
\hline & 8 & -2034.691988 & -2034.691985 & -2062.617031 & -2062.617078 \\
\hline & 9 & -2034.684896 & -2034.684889 & -2062.610776 & -2062.610754 \\
\hline & 10 & -2034.684896 & -2034.684889 & -2062.610776 & -2062.610754 \\
\hline & 1 & -2222.017395 & -2222.017358 & -2254.071415 & -2254.071395 \\
\hline & 2 & -2222.017395 & -2222.017358 & -2254.071415 & -2254.071395 \\
\hline & 3 & -2221.940851 & -2221.941114 & -2254.007447 & -2254.008208 \\
\hline & 4 & -2221.940851 & -2221.941114 & -2254.007447 & -2254.008208 \\
\hline \multirow{10}{*}{$\mathrm{Cu}(\text { gly })_{2}$} & 5 & -2221.933947 & -2221.933928 & -2254.000124 & -2254.000236 \\
\hline & 6 & -2221.933947 & -2221.933928 & -2254.000124 & -2254.000236 \\
\hline & 7 & -2221.925756 & -2221.925640 & -2253.994512 & -2253.993941 \\
\hline & 8 & -2221.925756 & -2221.925640 & -2253.994512 & -2253.993941 \\
\hline & 9 & -2221.918679 & -2221.918571 & -2253.987139 & -2253.986714 \\
\hline & 10 & -2221.918679 & -2221.918571 & -2253.987139 & -2253.986714 \\
\hline & 1 & -2607.104192 & -2607.104147 & -2652.280961 & -2652.281011 \\
\hline & 2 & -2607.104192 & -2607.104147 & -2652.280961 & -2652.281011 \\
\hline & 3 & -2607.026959 & -2607.027212 & -2652.216921 & -2652.217449 \\
\hline & 4 & -2607.026959 & -2607.027212 & -2652.216921 & -2652.217449 \\
\hline \multirow{10}{*}{$\mathrm{Cu}(\mathrm{ox})_{2}$} & 5 & -2607.021679 & -2607.021647 & -2652.211501 & -2652.211461 \\
\hline & 6 & -2607.021679 & -2607.021647 & -2652.211501 & -2652.211461 \\
\hline & 7 & -2607.015853 & -2607.015806 & -2652.207310 & -2652.207021 \\
\hline & 8 & -2607.015853 & -2607.015806 & -2652.207310 & -2652.207021 \\
\hline & 9 & -2607.008809 & -2607.008716 & -2652.200374 & -2652.200082 \\
\hline & 10 & -2607.008809 & -2607.008716 & -2652.200374 & -2652.200082 \\
\hline & 1 & -2454.743661 & -2454.743443 & -2495.155288 & -2495.155034 \\
\hline & 2 & -2454.743661 & -2454.743443 & -2495.155288 & -2495.155034 \\
\hline & 3 & -2454.664863 & -2454.664866 & -2495.089370 & -2495.089491 \\
\hline & 4 & -2454.664863 & -2454.664866 & -2495.089370 & -2495.089491 \\
\hline \multirow{10}{*}{$\mathrm{Cu}(\mathrm{sac})_{2}$} & 5 & -2454.658334 & -2454.657991 & -2495.083068 & -2495.083245 \\
\hline & 6 & -2454.658334 & -2454.657991 & -2495.083068 & -2495.083245 \\
\hline & 7 & -2454.654370 & -2454.654781 & -2495.080002 & -2495.080091 \\
\hline & 8 & -2454.654370 & -2454.654781 & -2495.080002 & -2495.080091 \\
\hline & 9 & -2454.646899 & -2454.647153 & -2495.073165 & -2495.073237 \\
\hline & 10 & -2454.646899 & -2454.647153 & -2495.073165 & -2495.073237 \\
\hline & 1 & -2344.587163 & -2344.587163 & -2381.166514 & -2381.166514 \\
\hline & 2 & -2344.587163 & -2344.587163 & -2381.166514 & -2381.166514 \\
\hline & 3 & -2344.518945 & -2344.518941 & -2381.111633 & -2381.111698 \\
\hline & 4 & -2344.518945 & -2344.518941 & -2381.111633 & -2381.111698 \\
\hline \multirow{7}{*}{$\mathrm{Cu}(\mathrm{acac})_{2}$} & 5 & -2344.507450 & -2344.507451 & -2381.101654 & -2381.101632 \\
\hline & 6 & -2344.507450 & -2344.507451 & -2381.101654 & -2381.101632 \\
\hline & 7 & -2344.506083 & -2344.506083 & -2381.099836 & -2381.099816 \\
\hline & 8 & -2344.506083 & -2344.506083 & -2381.099836 & -2381.099816 \\
\hline & 9 & -2344.497402 & -2344.497407 & -2381.092524 & -2381.092483 \\
\hline & 10 & -2344.497402 & -2344.497407 & -2381.092524 & -2381.092483 \\
\hline & 1 & -2937.057047 & -2937.057048 & -2979.270478 & -2979.270481 \\
\hline \multirow[t]{2}{*}{$\mathrm{Cu}(\mathrm{kts})_{2}$} & 2 & -2937.057047 & -2937.057048 & -2979.270478 & -2979.270481 \\
\hline & 3 & -2936.975241 & -2936.975339 & -2979.197323 & -2979.197449 \\
\hline
\end{tabular}




\begin{tabular}{|c|c|c|c|c|c|}
\hline & 4 & -2936.975241 & -2936.975339 & -2979.197323 & -2979.197449 \\
\hline & 5 & -2936.971884 & -2936.971775 & -2979.194407 & -2979.194198 \\
\hline & 6 & -2936.971884 & -2936.971775 & -2979.194407 & -2979.194198 \\
\hline & 7 & -2936.968982 & -2936.969077 & -2979.191124 & -2979.191258 \\
\hline & 8 & -2936.968982 & -2936.969077 & -2979.191124 & -2979.191258 \\
\hline & 9 & -2936.962463 & -2936.962378 & -2979.184973 & -2979.184925 \\
\hline & 10 & -2936.962463 & -2936.962378 & -2979.184973 & -2979.184925 \\
\hline \multirow{10}{*}{$\mathrm{Cu}(\mathrm{dtc})_{2}$} & 1 & -3753.277976 & -3753.277978 & -3809.433590 & -3809.433596 \\
\hline & 2 & -3753.277976 & -3753.277978 & -3809.433590 & -3809.433596 \\
\hline & 3 & -3753.199671 & -3753.199767 & -3809.358465 & -3809.358763 \\
\hline & 4 & -3753.199671 & -3753.199767 & -3809.358465 & -3809.358763 \\
\hline & 5 & -3753.197367 & -3753.197240 & -3809.356726 & -3809.356389 \\
\hline & 6 & -3753.197367 & -3753.197240 & -3809.356726 & -3809.356389 \\
\hline & 7 & -3753.193635 & -3753.193765 & -3809.352733 & -3809.353031 \\
\hline & 8 & -3753.193635 & -3753.193765 & -3809.352733 & -3809.353031 \\
\hline & 9 & -3753.188226 & -3753.188088 & -3809.347765 & -3809.347480 \\
\hline & 10 & -3753.188226 & -3753.188088 & -3809.347765 & -3809.347480 \\
\hline \multirow{10}{*}[\mathrm{Ni}(\text{cyclam})]{$^{+}$} & 1 & -2134.245141 & -2134.24514296 & -2168.987301 & -2168.987304 \\
\hline & 2 & -2134.245141 & -2134.24514296 & -2168.987301 & -2168.987304 \\
\hline & 3 & -2134.177309 & -2134.17745002 & -2168.932964 & -2168.933118 \\
\hline & 4 & -2134.177309 & -2134.17745002 & -2168.932964 & -2168.933118 \\
\hline & 5 & -2134.166946 & -2134.16685337 & -2168.922264 & -2168.922215 \\
\hline & 6 & -2134.166946 & -2134.16685337 & -2168.922264 & -2168.922215 \\
\hline & 7 & -2134.164264 & -2134.16426797 & -2168.920632 & -2168.920651 \\
\hline & 8 & -2134.164264 & -2134.16426797 & -2168.920632 & -2168.920651 \\
\hline & 9 & -2134.152933 & -2134.15293233 & -2168.914199 & -2168.914127 \\
\hline & 10 & -2134.152933 & -2134.15293233 & -2168.914199 & -2168.914127 \\
\hline \multirow{10}{*}{$\mathrm{NiH}(\mathrm{CO})_{3}$} & 1 & -1860.835668 & -1860.835669 & -1885.185577 & -1885.185576 \\
\hline & 2 & -1860.835668 & -1860.835669 & -1885.185577 & -1885.185576 \\
\hline & 3 & -1860.775631 & -1860.776118 & -1885.122400 & -1885.122609 \\
\hline & 4 & -1860.775631 & -1860.776118 & -1885.122400 & -1885.122609 \\
\hline & 5 & -1860.773952 & -1860.774185 & -1885.120377 & -1885.120523 \\
\hline & 6 & -1860.773952 & -1860.774185 & -1885.120377 & -1885.120523 \\
\hline & 7 & -1860.768048 & -1860.767913 & -1885.113719 & -1885.113903 \\
\hline & 8 & -1860.768048 & -1860.767913 & -1885.113719 & -1885.113903 \\
\hline & 9 & -1860.767586 & -1860.767377 & -1885.113592 & -1885.113526 \\
\hline & 10 & -1860.767586 & -1860.767377 & -1885.113592 & -1885.113526 \\
\hline
\end{tabular}


Table S5.2. Absolute energies (in hartree) for $\mathrm{d}^{7}$ configurations at CASSCF, CASPT2, XMSCASPT2, MC-PDFT and XMS-PDFT levels. An IPEA shift of 0.25 a.u. and an imaginary shift of 0.3 a.u. are used for CASPT2 and XMS-CASPT2.

\begin{tabular}{|c|c|c|c|c|}
\hline Molecule & Spin-orbit state & $\mathrm{SA}(5)-\mathrm{CASSCF}$ & CASPT2 & XMS-CASPT2 \\
\hline \multirow{10}{*}{ Co(acacen) } & 1 & -2116.182743 & -2119.541507 & -2119.547968 \\
\hline & 2 & -2116.182743 & -2119.541507 & -2119.547968 \\
\hline & 3 & -2116.179181 & -2119.534180 & -2119.541039 \\
\hline & 4 & -2116.179181 & -2119.534180 & -2119.541039 \\
\hline & 5 & -2116.171876 & -2119.528144 & -2119.534988 \\
\hline & 6 & -2116.171876 & -2119.528144 & -2119.534988 \\
\hline & 7 & -2116.136460 & -2119.503166 & -2119.505024 \\
\hline & 8 & -2116.136460 & -2119.503166 & -2119.505024 \\
\hline & 9 & -2116.085955 & -2119.448686 & -2119.451316 \\
\hline & 10 & -2116.085955 & -2119.448686 & -2119.451316 \\
\hline \multirow{10}{*}{$\mathrm{Co}(\operatorname{sacsac})_{2}$} & 1 & -3373.661054 & -3376.524223 & -3376.539213 \\
\hline & 2 & -3373.661054 & -3376.524223 & -3376.539213 \\
\hline & 3 & -3373.657031 & -3376.518924 & -3376.533057 \\
\hline & 4 & -3373.657031 & -3376.518924 & -3376.533057 \\
\hline & 5 & -3373.642213 & -3376.502477 & -3376.517936 \\
\hline & 6 & -3373.642213 & -3376.502477 & -3376.517936 \\
\hline & 7 & -3373.602481 & -3376.472959 & -3376.485241 \\
\hline & 8 & -3373.602481 & -3376.472959 & -3376.485241 \\
\hline & 9 & -3373.551224 & -3376.420423 & -3376.440444 \\
\hline & 10 & -3373.551224 & -3376.420423 & -3376.440444 \\
\hline \multirow{10}{*}{ Co(tacacen) } & 1 & -2763.525084 & -2766.789009 & -2766.803401 \\
\hline & 2 & -2763.525084 & -2766.789009 & -2766.803401 \\
\hline & 3 & -2763.521332 & -2766.784223 & -2766.796266 \\
\hline & 4 & -2763.521332 & -2766.784223 & -2766.796266 \\
\hline & 5 & -2763.509713 & -2766.772193 & -2766.785854 \\
\hline & 6 & -2763.509713 & -2766.772193 & -2766.785854 \\
\hline & 7 & -2763.471838 & -2766.743779 & -2766.754742 \\
\hline & 8 & -2763.471838 & -2766.743779 & -2766.754742 \\
\hline & 9 & -2763.420321 & -2766.689824 & -2766.706861 \\
\hline & 10 & -2763.420321 & -2766.689824 & -2766.706861 \\
\hline \multirow{10}{*}[\mathrm{Ni}(\mathrm{mnt})_{2}]{$^{-}$} & 1 & -3635.015073 & -3638.273501 & -3638.291741 \\
\hline & 2 & -3635.015073 & -3638.273501 & -3638.291741 \\
\hline & 3 & -3635.011206 & -3638.266318 & -3638.282235 \\
\hline & 4 & -3635.011206 & -3638.266318 & -3638.282235 \\
\hline & 5 & -3635.003821 & -3638.260045 & -3638.278611 \\
\hline & 6 & -3635.003821 & -3638.260045 & -3638.278611 \\
\hline & 7 & -3634.963506 & -3638.227449 & -3638.239895 \\
\hline & 8 & -3634.963506 & -3638.227449 & -3638.239895 \\
\hline & 9 & -3634.884959 & -3638.139584 & -3638.163784 \\
\hline & 10 & -3634.884959 & -3638.139584 & -3638.163784 \\
\hline
\end{tabular}




\begin{tabular}{|c|c|c|c|c|c|}
\hline Molecule & $\begin{array}{l}\text { Spin- } \\
\text { orbit } \\
\text { state }\end{array}$ & $\begin{array}{c}\text { MC-PDFT } \\
\text { (tPBE) }\end{array}$ & $\begin{array}{l}\text { XMS-PDFT } \\
(\mathrm{tPBE})\end{array}$ & $\begin{array}{c}\text { MC-PDFT } \\
\text { (tPBE-HLE) }\end{array}$ & $\begin{array}{l}\text { XMS-PDFT } \\
\text { (tPBE-HLE) }\end{array}$ \\
\hline \multirow{10}{*}{ Co(acacen) } & 1 & -2120.724369 & -2120.724042 & -2157.304687 & -2157.302487 \\
\hline & 2 & -2120.724369 & -2120.724042 & -2157.304687 & -2157.302487 \\
\hline & 3 & -2120.720670 & -2120.720425 & -2157.292227 & -2157.292156 \\
\hline & 4 & -2120.720670 & -2120.720425 & -2157.292227 & -2157.292156 \\
\hline & 5 & -2120.709287 & -2120.709254 & -2157.282519 & -2157.282518 \\
\hline & 6 & -2120.709287 & -2120.709254 & -2157.282519 & -2157.282518 \\
\hline & 7 & -2120.677198 & -2120.676825 & -2157.241245 & -2157.242246 \\
\hline & 8 & -2120.677198 & -2120.676825 & -2157.241245 & -2157.242246 \\
\hline & 9 & -2120.626677 & -2120.626680 & -2157.210727 & -2157.210743 \\
\hline & 10 & -2120.626677 & -2120.626680 & -2157.210727 & -2157.210743 \\
\hline \multirow{10}{*}{$\mathrm{Co}(\mathrm{sacsac})_{2}$} & 1 & -3378.766116 & -3378.765046 & -3429.528425 & -3429.526684 \\
\hline & 2 & -3378.766116 & -3378.765046 & -3429.528425 & -3429.526684 \\
\hline & 3 & -3378.760681 & -3378.760844 & -3429.517322 & -3429.517234 \\
\hline & 4 & -3378.760681 & -3378.760844 & -3429.517322 & -3429.517234 \\
\hline & 5 & -3378.741157 & -3378.740958 & -3429.500090 & -3429.500224 \\
\hline & 6 & -3378.741157 & -3378.740958 & -3429.500090 & -3429.500224 \\
\hline & 7 & -3378.705693 & -3378.705852 & -3429.458884 & -3429.459596 \\
\hline & 8 & -3378.705693 & -3378.705852 & -3429.458884 & -3429.459596 \\
\hline & 9 & -3378.661521 & -3378.661546 & -3429.430789 & -3429.430781 \\
\hline & 10 & -3378.661521 & -3378.661546 & -3429.430789 & -3429.430781 \\
\hline \multirow{10}{*}{ Co(tacacen) } & 1 & -2768.528860 & -2768.528791 & -2813.140921 & -2813.141450 \\
\hline & 2 & -2768.528860 & -2768.528791 & -2813.140921 & -2813.141450 \\
\hline & 3 & -2768.524985 & -2768.525098 & -2813.134874 & -2813.136013 \\
\hline & 4 & -2768.524985 & -2768.525098 & -2813.134874 & -2813.136013 \\
\hline & 5 & -2768.508057 & -2768.508035 & -2813.117352 & -2813.117389 \\
\hline & 6 & -2768.508057 & -2768.508035 & -2813.117352 & -2813.117389 \\
\hline & 7 & -2768.473584 & -2768.474333 & -2813.075881 & -2813.076654 \\
\hline & 8 & -2768.473584 & -2768.474333 & -2813.075881 & -2813.076654 \\
\hline & 9 & -2768.425428 & -2768.425427 & -2813.044302 & -2813.044326 \\
\hline & 10 & -2768.425428 & -2768.425427 & -2813.044302 & -2813.044326 \\
\hline \multirow{10}{*}[\mathrm{Ni}(\mathrm{mnt})_{2}]{$^{-}$} & 1 & -3640.527713 & -3640.526249 & -3694.957107 & -3694.954189 \\
\hline & 2 & -3640.527713 & -3640.526249 & -3694.957107 & -3694.954189 \\
\hline & 3 & -3640.523481 & -3640.524519 & -3694.949863 & -3694.949416 \\
\hline & 4 & -3640.523481 & -3640.524519 & -3694.949863 & -3694.949416 \\
\hline & 5 & -3640.514331 & -3640.514150 & -3694.940826 & -3694.940636 \\
\hline & 6 & -3640.514331 & -3640.514150 & -3694.940826 & -3694.940636 \\
\hline & 7 & -3640.478273 & -3640.476207 & -3694.902947 & -3694.902821 \\
\hline & 8 & -3640.478273 & -3640.476207 & -3694.902947 & -3694.902821 \\
\hline & 9 & -3640.392099 & -3640.392108 & -3694.832046 & -3694.832041 \\
\hline & 10 & -3640.392099 & -3640.392108 & -3694.832046 & -3694.832041 \\
\hline
\end{tabular}


Table S5.3. Absolute energies (in hartree) for $\mathrm{d}^{5}$ configurations at CASSCF, CASPT2, XMSCASPT2, MC-PDFT and XMS-PDFT levels. An IPEA shift of 0.25 a.u. and an imaginary shift of 0.3 a.u. are used for CASPT2 and XMS-CASPT2.

\begin{tabular}{|c|c|c|c|c|c|c|}
\hline Molecule & $\begin{array}{l}\text { Spin- } \\
\text { orbit } \\
\text { state }\end{array}$ & $\mathrm{SA}(5)-\mathrm{CASSCF}$ & $\begin{array}{l}\text { MC-PDFT } \\
\text { (tPBE) }\end{array}$ & $\begin{array}{l}\text { XMS-PDFT } \\
\text { (tPBE) }\end{array}$ & $\begin{array}{l}\text { MC-PDFT } \\
\text { (tPBE-HLE) }\end{array}$ & $\begin{array}{l}\text { XMS-PDFT } \\
\text { (tPBE-HLE) }\end{array}$ \\
\hline \multirow{10}{*}[\mathrm{Fe}(\mathrm{bpy})3]{$^{3+}$} & 1 & -2748.570088 & -2756.844515 & -2756.841978 & -2816.514786 & -2816.511871 \\
\hline & 2 & -2748.570088 & -2756.844515 & -2756.841978 & -2816.514786 & -2816.511871 \\
\hline & 3 & -2748.563927 & -2756.837043 & -2756.836231 & -2816.507302 & -2816.506181 \\
\hline & 4 & -2748.563927 & -2756.837043 & -2756.836231 & -2816.507302 & -2816.506181 \\
\hline & 5 & -2748.562414 & -2756.835334 & -2756.834366 & -2816.505530 & -2816.503687 \\
\hline & 6 & -2748.562414 & -2756.835334 & -2756.834366 & -2816.505530 & -2816.503687 \\
\hline & 7 & -2748.476855 & -2756.735386 & -2756.735321 & -2816.406593 & -2816.406414 \\
\hline & 8 & -2748.476855 & -2756.735386 & -2756.735321 & -2816.406593 & -2816.406414 \\
\hline & 9 & -2748.475457 & -2756.734304 & -2756.734191 & -2816.405502 & -2816.405368 \\
\hline & 10 & -2748.475457 & -2756.734304 & -2756.734191 & -2816.405502 & -2816.405368 \\
\hline \multirow{10}{*}{$\begin{array}{l}{\left[\mathrm{Fe}\left(\mathrm{N}_{3}\right)\right.} \\
(\mathrm{MePy} 2 \mathrm{tacn})]^{2+}\end{array}$} & 1 & -2442.645962 & -2449.446913 & -2449.448210 & -2499.318489 & -2499.318363 \\
\hline & 2 & -2442.645962 & -2449.446913 & -2449.448210 & -2499.318489 & -2499.318363 \\
\hline & 3 & -2442.641903 & -2449.441318 & -2449.442131 & -2499.313446 & -2499.314495 \\
\hline & 4 & -2442.641903 & -2449.441318 & -2449.442131 & -2499.313446 & -2499.314495 \\
\hline & 5 & -2442.636674 & -2449.433984 & -2449.437223 & -2499.304741 & -2499.303419 \\
\hline & 6 & -2442.636674 & -2449.433984 & -2449.437223 & -2499.304741 & -2499.303419 \\
\hline & 7 & -2442.559257 & -2449.354119 & -2449.354840 & -2499.233111 & -2499.231908 \\
\hline & 8 & -2442.559257 & -2449.354119 & -2449.354840 & -2499.233111 & -2499.231908 \\
\hline & 9 & -2442.556086 & -2449.350128 & -2449.350943 & -2499.229856 & -2499.228541 \\
\hline & 10 & -2442.556086 & -2449.350128 & -2449.350943 & -2499.229856 & -2499.228541 \\
\hline \multirow{10}{*}[\mathrm{Fe}(\mathrm{PyMS})_{2}]{$^{+}$} & 1 & -3059.602702 & -3066.633674 & -3066.634815 & -3123.456896 & -3123.455857 \\
\hline & 2 & -3059.602702 & -3066.633674 & -3066.634815 & -3123.456896 & -3123.455857 \\
\hline & 3 & -3059.583239 & -3066.611227 & -3066.608877 & -3123.437467 & -3123.433749 \\
\hline & 4 & -3059.583239 & -3066.611227 & -3066.608877 & -3123.437467 & -3123.433749 \\
\hline & 5 & -3059.577424 & -3066.605583 & -3066.603348 & -3123.432279 & -3123.428046 \\
\hline & 6 & -3059.577424 & -3066.605583 & -3066.603348 & -3123.432279 & -3123.428046 \\
\hline & 7 & -3059.500779 & -3066.531334 & -3066.528089 & -3123.363181 & -3123.357372 \\
\hline & 8 & -3059.500779 & -3066.531334 & -3066.528089 & -3123.363181 & -3123.357372 \\
\hline & 9 & -3059.499100 & -3066.529910 & -3066.526453 & -3123.362066 & -3123.355730 \\
\hline & 10 & -3059.499100 & -3066.529910 & -3066.526453 & -3123.362066 & -3123.355730 \\
\hline \multirow{10}{*}[\mathrm{Fe}(\mathrm{PyAS})_{2}]{$^{+}$} & 1 & -3206.538622 & -3214.209447 & -3214.208788 & -3275.222168 & -3275.221631 \\
\hline & 2 & -3206.538622 & -3214.209447 & -3214.208788 & -3275.222168 & -3275.221631 \\
\hline & 3 & -3206.521571 & -3214.191162 & -3214.190833 & -3275.205809 & -3275.205207 \\
\hline & 4 & -3206.521571 & -3214.191162 & -3214.190833 & -3275.205809 & -3275.205207 \\
\hline & 5 & -3206.517645 & -3214.187161 & -3214.186653 & -3275.202343 & -3275.201329 \\
\hline & 6 & -3206.517645 & -3214.187161 & -3214.186653 & -3275.202343 & -3275.201329 \\
\hline & 7 & -3206.435173 & -3214.100545 & -3214.103077 & -3275.116711 & -3275.113539 \\
\hline & 8 & -3206.435173 & -3214.100545 & -3214.103077 & -3275.116711 & -3275.113539 \\
\hline & 9 & -3206.434356 & -3214.099727 & -3214.102256 & -3275.115856 & -3275.112678 \\
\hline & 10 & -3206.434356 & -3214.099727 & -3214.102256 & -3275.115856 & -3275.112678 \\
\hline \multirow{10}{*}{$\begin{array}{l}\operatorname{Mn}(\text { dmpe }) 2 \\
\text { (o-xylylene) }\end{array}$} & 1 & -3304.851995 & -3312.012335 & -3312.012956 & -3372.487801 & -3372.488229 \\
\hline & 2 & -3304.851995 & -3312.012335 & -3312.012956 & -3372.487801 & -3372.488229 \\
\hline & 3 & -3304.828514 & -3311.988713 & -3311.988675 & -3372.466499 & -3372.467113 \\
\hline & 4 & -3304.828514 & -3311.988713 & -3311.988675 & -3372.466499 & -3372.467113 \\
\hline & 5 & -3304.824218 & -3311.985405 & -3311.985358 & -3372.462778 & -3372.462987 \\
\hline & 6 & -3304.824218 & -3311.985405 & -3311.985358 & -3372.462778 & -3372.462987 \\
\hline & 7 & -3304.717687 & -3311.883265 & -3311.882481 & -3372.376241 & -3372.374086 \\
\hline & 8 & -3304.717687 & -3311.883265 & -3311.882481 & -3372.376241 & -3372.374086 \\
\hline & 9 & -3304.714335 & -3311.877813 & -3311.878674 & -3372.369420 & -3372.369369 \\
\hline & 10 & -3304.714335 & -3311.877813 & -3311.878674 & -3372.369420 & -3372.369369 \\
\hline
\end{tabular}


Table S5.4. Absolute energies (in hartree) for $\mathrm{d}^{1}$ configurations at CASSCF, CASPT2, XMSCASPT2, MC-PDFT and XMS-PDFT levels. An IPEA shift of 0.25 a.u. and an imaginary shift of 0.3 a.u. are used for CASPT2 and XMS-CASPT2.

\begin{tabular}{|c|c|c|c|c|}
\hline Molecule & Spin-orbit state & SA(5)-CASSCF & CASPT2 & XMS-CASPT2 \\
\hline \multirow{11}{*}[\mathrm{MnN}(\mathrm{CN})_{4}]{$^{-}$} & 1 & -1581.33717 & -1583.52029 & -1583.53065 \\
\hline & 2 & -1581.33717 & -1583.52029 & -1583.53065 \\
\hline & 3 & -1581.20724 & -1583.38802 & -1583.40240 \\
\hline & 4 & -1581.20724 & -1583.38802 & -1583.40240 \\
\hline & 5 & -1581.20664 & -1583.38742 & -1583.40180 \\
\hline & 6 & -1581.20664 & -1583.38742 & -1583.40180 \\
\hline & 7 & -1581.17084 & -1583.34197 & -1583.34402 \\
\hline & 8 & -1581.17084 & -1583.34197 & -1583.34402 \\
\hline & 9 & -1581.16655 & -1583.32884 & -1583.34122 \\
\hline & 10 & -1581.16655 & -1583.32884 & -1583.34122 \\
\hline & 1 & -1953.82554 & -1957.70436 & -1957.71469 \\
\hline \multirow{9}{*}[\mathrm{MnN}(\text{cyclam})(\mathrm{NCCH}_{3})]{$^{3+}$} & 2 & -1953.82554 & -1957.70436 & -1957.71469 \\
\hline & 3 & -1953.70378 & -1957.57541 & -1957.58939 \\
\hline & 4 & -1953.70378 & -1957.57541 & -1957.58939 \\
\hline & 5 & -1953.69875 & -1957.57430 & -1957.58791 \\
\hline & 6 & -1953.69875 & -1957.57430 & -1957.58791 \\
\hline & 7 & -1953.69725 & -1957.56333 & -1957.57225 \\
\hline & 8 & -1953.69725 & -1957.56333 & -1957.57225 \\
\hline & 9 & -1953.67006 & -1957.53584 & -1957.53553 \\
\hline & 10 & -1953.67006 & -1957.53584 & -1957.53553 \\
\hline \multirow{10}{*}{$\mathrm{VO}(\mathrm{acac})_{2}$} & 1 & -1710.17312 & -1713.51506 & -1713.54060 \\
\hline & 2 & -1710.17312 & -1713.51506 & -1713.54060 \\
\hline & 3 & -1710.10531 & -1713.43478 & -1713.45551 \\
\hline & 4 & -1710.10531 & -1713.43478 & -1713.45551 \\
\hline & 5 & -1710.10323 & -1713.42790 & -1713.43305 \\
\hline & 6 & -1710.10323 & -1713.42790 & -1713.43305 \\
\hline & 7 & -1710.10157 & -1713.42468 & -1713.43021 \\
\hline & 8 & -1710.10157 & -1713.42468 & -1713.43021 \\
\hline & 9 & -1710.04553 & -1713.36574 & -1713.37033 \\
\hline & 10 & -1710.04553 & -1713.36574 & -1713.37033 \\
\hline \multirow{10}{*}{$\mathrm{VO}\left(\mathrm{H}_{2} \mathrm{O}\right) 5^{2+}$} & 1 & -1403.23233 & -1405.29939 & -1405.32963 \\
\hline & 2 & -1403.23233 & -1405.29939 & -1405.32963 \\
\hline & 3 & -1403.16966 & -1405.22735 & -1405.25498 \\
\hline & 4 & -1403.16966 & -1405.22735 & -1405.25498 \\
\hline & 5 & -1403.16171 & -1405.20111 & -1405.20382 \\
\hline & 6 & -1403.16171 & -1405.20111 & -1405.20382 \\
\hline & 7 & -1403.15958 & -1405.19945 & -1405.20253 \\
\hline & 8 & -1403.15958 & -1405.19945 & -1405.20253 \\
\hline & 9 & -1403.06982 & -1405.10865 & -1405.11229 \\
\hline & 10 & -1403.06982 & -1405.10865 & -1405.11229 \\
\hline
\end{tabular}

\begin{tabular}{cccccc}
\hline Molecule & $\begin{array}{c}\text { Spin- } \\
\text { orbit } \\
\text { state }\end{array}$ & $\begin{array}{c}\text { MC-PDFT } \\
\text { (tPBE) }\end{array}$ & $\begin{array}{c}\text { XMS-PDFT } \\
\text { (tPBE) }\end{array}$ & $\begin{array}{c}\text { MC-PDFT } \\
\text { (tPBE-HLE) }\end{array}$ & $\begin{array}{c}\text { XMS-PDFT } \\
\text { (tPBE-HLE) }\end{array}$ \\
\hline & 1 & -1584.288229 & -1584.282462 & -1608.962293 & -1608.961230 \\
& 2 & -1584.288229 & -1584.282462 & -1608.962293 & -1608.961230 \\
{$[\mathrm{MnN}(\mathrm{CN}) 4]^{-}$} & 3 & -1584.174778 & -1584.174776 & -1608.847903 & -1608.847897 \\
& 4 & -1584.174778 & -1584.174776 & -1608.847903 & -1608.847897 \\
& 5 & -1584.174180 & -1584.174179 & -1608.847305 & -1608.847299 \\
& 6 & -1584.174180 & -1584.174179 & -1608.847305 & -1608.847299 \\
& 7 & -1584.117442 & -1584.127372 & -1608.805149 & -1608.803649 \\
& 8 & -1584.117442 & -1584.127372 & -1608.805149 & -1608.803649 \\
& 9 & -1584.099450 & -1584.099448 & -1608.785974 & -1608.785974 \\
& 10 & -1584.099450 & -1584.099448 & -1608.785974 & -1608.785974 \\
& 1 & -1958.810130 & -1958.803448 & -1996.357169 & -1996.355559 \\
& 2 & -1958.810130 & -1958.803448 & -1996.357169 & -1996.355559
\end{tabular}


$\mathrm{VO}(\mathrm{acac})_{2}$

\begin{tabular}{ccccc}
3 & -1958.701871 & -1958.701818 & -1996.245812 & -1996.245806 \\
4 & -1958.701871 & -1958.701818 & -1996.245812 & -1996.245806 \\
5 & -1958.700473 & -1958.700376 & -1996.244448 & -1996.244269 \\
6 & -1958.700473 & -1958.700376 & -1996.244448 & -1996.244269 \\
7 & -1958.671254 & -1958.671302 & -1996.231071 & -1996.230931 \\
8 & -1958.671254 & -1958.671302 & -1996.231071 & -1996.230931 \\
9 & -1958.649745 & -1958.661951 & -1996.203642 & -1996.201819 \\
10 & -1958.649745 & -1958.661951 & -1996.203642 & -1996.201819 \\
1 & -1714.553398 & -1714.553388 & -1747.732129 & -1747.732119 \\
2 & -1714.553398 & -1714.553388 & -1747.732129 & -1747.732119 \\
3 & -1714.472093 & -1714.472088 & -1747.668010 & -1747.667988 \\
4 & -1714.472093 & -1714.472088 & -1747.668010 & -1747.667988 \\
5 & -1714.466952 & -1714.466951 & -1747.651869 & -1747.651867 \\
6 & -1714.466952 & -1714.466951 & -1747.651869 & -1747.651867 \\
7 & -1714.464624 & -1714.464625 & -1747.650081 & -1747.650083 \\
8 & -1714.464624 & -1714.464625 & -1747.650081 & -1747.650083 \\
9 & -1714.398766 & -1714.398799 & -1747.576501 & -1747.576547 \\
10 & -1714.398766 & -1714.398799 & -1747.576501 & -1747.576547 \\
1 & -1405.983367 & -1405.983346 & -1428.774224 & -1428.774201 \\
2 & -1405.983367 & -1405.983346 & -1428.774224 & -1428.774201 \\
3 & -1405.911936 & -1405.911941 & -1428.720070 & -1428.720068 \\
4 & -1405.911936 & -1405.911941 & -1428.720070 & -1428.720068 \\
5 & -1405.887129 & -1405.887120 & -1428.682497 & -1428.682524 \\
6 & -1405.887129 & -1405.887120 & -1428.682497 & -1428.682524 \\
7 & -1405.885915 & -1405.885914 & -1428.681413 & -1428.681373 \\
8 & -1405.885915 & -1405.885914 & -1428.681413 & -1428.681373 \\
9 & -1405.792311 & -1405.792347 & -1428.592485 & -1428.592527 \\
10 & -1405.792311 & -1405.792347 & -1428.592485 & -1428.592527 \\
\hline & & & &
\end{tabular}


Table S5.5. Absolute energies (in hartree) for $\mathrm{d}^{9}$ configurations at CASCI, MC-PDFT and XMSPDFT levels with CASCI scheme.

\begin{tabular}{|c|c|c|c|c|c|c|}
\hline Molecule & $\begin{array}{l}\text { Spin-orbit } \\
\text { state }\end{array}$ & CASCI $(1,10)$ & $\begin{array}{c}\text { MC(1,10)- } \\
\text { PDFT (tPBE) }\end{array}$ & $\begin{array}{l}\text { XMS }(1,10)- \\
\text { PDFT (tPBE) }\end{array}$ & $\begin{array}{l}\mathrm{MC}(1,10)-\mathrm{PDFT} \\
\quad(\mathrm{tPBE}-\mathrm{HLE})\end{array}$ & $\begin{array}{c}\text { XMS }(1,10)- \\
\text { PDFT (tPBE- } \\
\text { HLE) }\end{array}$ \\
\hline \multirow{19}{*}[\mathrm{Cu}(\mathrm{NH}_{3})4]{$^{2+}$} & 1 & -1877.919223 & -1880.087585 & -1880.087371 & -1902.936698 & -1902.994158 \\
\hline & 2 & -1877.919223 & -1880.087585 & -1880.087371 & -1902.936698 & -1902.994158 \\
\hline & 3 & -1877.854880 & -1880.010380 & -1880.010421 & -1902.869826 & -1902.869917 \\
\hline & 4 & -1877.854880 & -1880.010380 & -1880.010421 & -1902.869826 & -1902.869917 \\
\hline & 5 & -1877.840007 & -1879.994263 & -1879.994299 & -1902.855047 & -1902.855116 \\
\hline & 6 & -1877.840007 & -1879.994263 & -1879.994299 & -1902.855047 & -1902.855116 \\
\hline & 7 & -1877.838001 & -1879.992082 & -1879.992108 & -1902.853105 & -1902.853119 \\
\hline & 8 & -1877.838001 & -1879.992082 & -1879.992108 & -1902.853105 & -1902.853119 \\
\hline & 9 & -1877.818323 & -1879.970474 & -1879.970521 & -1902.834416 & -1902.834359 \\
\hline & 10 & -1877.818323 & -1879.970474 & -1879.970521 & -1902.834416 & -1902.834359 \\
\hline & 11 & -1877.569499 & -1879.833821 & -1879.795821 & -1902.713235 & -1902.693702 \\
\hline & 12 & -1877.569499 & -1879.833821 & -1879.795821 & -1902.713235 & -1902.693702 \\
\hline & 13 & -1875.884482 & -1878.195595 & -1878.195579 & -1901.039025 & -1901.039598 \\
\hline & 14 & -1875.884482 & -1878.195595 & -1878.195579 & -1901.039025 & -1901.039598 \\
\hline & 15 & -1875.857842 & -1878.193933 & -1878.193338 & -1901.031529 & -1901.031573 \\
\hline & 16 & -1875.857842 & -1878.193933 & -1878.193338 & -1901.031529 & -1901.031573 \\
\hline & 17 & -1875.853268 & -1878.155748 & -1878.155584 & -1900.996391 & -1900.996172 \\
\hline & 18 & -1875.853268 & -1878.155748 & -1878.155584 & -1900.996391 & -1900.996172 \\
\hline & 19 & -1875.850340 & -1878.151925 & -1878.151757 & -1900.992608 & -1900.992390 \\
\hline \multirow{21}{*}[\mathrm{Cu}(\mathrm{py})4]{$^{2+}$} & 20 & -1875.850340 & -1878.151925 & -1878.151757 & -1900.992608 & -1900.992390 \\
\hline & 1 & -2640.548523 & -2646.518488 & -2646.567693 & -2693.782382 & -2693.834722 \\
\hline & 2 & -2640.548523 & -2646.518488 & -2646.567693 & -2693.782382 & -2693.834722 \\
\hline & 3 & -2640.476834 & -2646.433018 & -2646.433063 & -2693.709584 & -2693.709642 \\
\hline & 4 & -2640.476834 & -2646.433018 & -2646.433063 & -2693.709584 & -2693.709642 \\
\hline & 5 & -2640.459543 & -2646.414714 & -2646.414946 & -2693.692774 & -2693.693066 \\
\hline & 6 & -2640.459543 & -2646.414714 & -2646.414946 & -2693.692774 & -2693.693066 \\
\hline & 7 & -2640.457297 & -2646.412340 & -2646.412543 & -2693.690609 & -2693.690857 \\
\hline & 8 & -2640.457297 & -2646.412340 & -2646.412543 & -2693.690609 & -2693.690857 \\
\hline & 9 & -2640.434943 & -2646.388018 & -2646.388002 & -2693.669669 & -2693.669620 \\
\hline & 10 & -2640.434943 & -2646.388018 & -2646.388002 & -2693.669669 & -2693.669620 \\
\hline & 11 & -2640.189535 & -2646.258567 & -2646.247985 & -2693.547261 & -2693.529017 \\
\hline & 12 & -2640.189535 & -2646.258567 & -2646.247985 & -2693.547261 & -2693.529017 \\
\hline & 13 & -2638.579123 & -2644.700249 & -2644.700262 & -2691.947220 & -2691.947275 \\
\hline & 14 & -2638.579123 & -2644.700249 & -2644.700262 & -2691.947220 & -2691.947275 \\
\hline & 15 & -2638.558054 & -2644.688432 & -2644.687655 & -2691.947038 & -2691.946225 \\
\hline & 16 & -2638.558054 & -2644.688432 & -2644.687655 & -2691.947038 & -2691.946225 \\
\hline & 17 & -2638.552953 & -2644.649018 & -2644.648830 & -2691.902633 & -2691.902377 \\
\hline & 18 & -2638.552953 & -2644.649018 & -2644.648830 & -2691.902633 & -2691.902377 \\
\hline & 19 & -2638.549702 & -2644.645070 & -2644.644878 & -2691.898727 & -2691.898468 \\
\hline & 20 & -2638.549702 & -2644.645070 & -2644.644878 & -2691.898727 & -2691.898468 \\
\hline \multirow{16}{*}[\mathrm{Cu}(\mathrm{iz})4]{$^{2+}$} & 1 & -2553.057757 & -2558.476481 & -2558.521873 & -2602.038959 & -2602.086283 \\
\hline & 2 & -2553.057757 & -2558.476481 & -2558.521873 & -2602.038959 & -2602.086283 \\
\hline & 3 & -2552.983276 & -2558.389652 & -2558.389689 & -2601.965379 & -2601.965428 \\
\hline & 4 & -2552.983276 & -2558.389652 & -2558.389689 & -2601.965379 & -2601.965428 \\
\hline & 5 & -2552.966896 & -2558.372253 & -2558.372298 & -2601.949386 & -2601.949448 \\
\hline & 6 & -2552.966896 & -2558.372253 & -2558.372298 & -2601.949386 & -2601.949448 \\
\hline & 7 & -2552.964656 & -2558.369890 & -2558.369939 & -2601.947259 & -2601.947291 \\
\hline & 8 & -2552.964656 & -2558.369890 & -2558.369939 & -2601.947259 & -2601.947291 \\
\hline & 9 & -2552.942720 & -2558.345981 & -2558.346040 & -2601.926862 & -2601.926950 \\
\hline & 10 & -2552.942720 & -2558.345981 & -2558.346040 & -2601.926862 & -2601.926950 \\
\hline & 11 & -2552.672845 & -2558.192490 & -2558.184990 & -2601.779491 & -2601.765541 \\
\hline & 12 & -2552.672845 & -2558.192490 & -2558.184990 & -2601.779491 & -2601.765541 \\
\hline & 13 & -2551.089986 & -2556.658862 & -2556.658828 & -2600.202958 & -2600.202881 \\
\hline & 14 & -2551.089986 & -2556.658862 & -2556.658828 & -2600.202958 & -2600.202881 \\
\hline & 15 & -2551.070254 & -2556.646038 & -2556.645245 & -2600.202483 & -2600.201648 \\
\hline & 16 & -2551.070254 & -2556.646038 & -2556.645245 & -2600.202483 & -2600.201648 \\
\hline
\end{tabular}




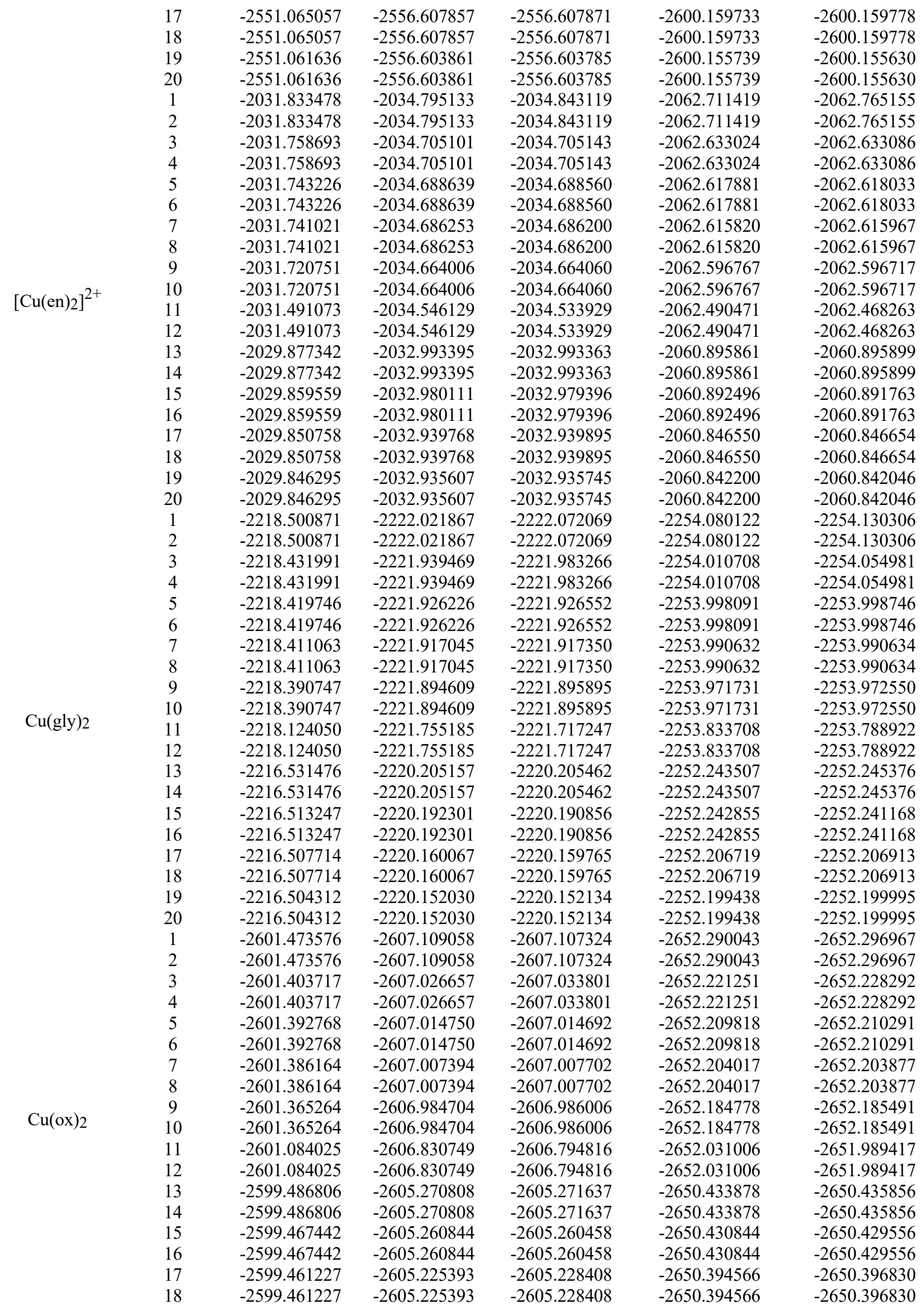




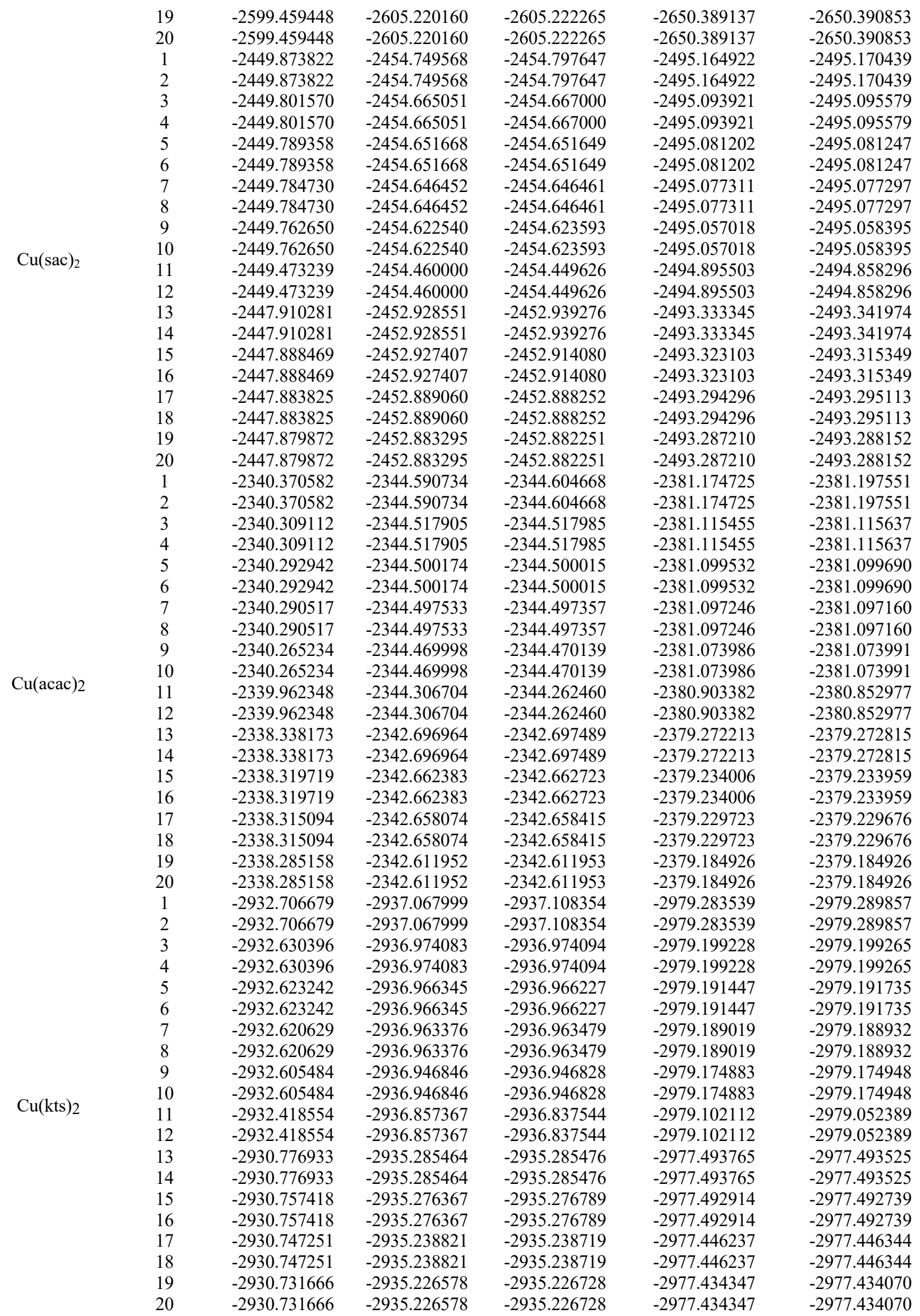




\begin{tabular}{|c|c|c|c|c|c|c|}
\hline & 1 & -3747.597246 & -3753.296696 & -3753.322745 & -3809.453530 & -3809.495177 \\
\hline & 2 & -3747.597246 & -3753.296696 & -3753.322745 & -3809.453530 & -3809.495177 \\
\hline & 3 & -3747.523469 & -3753.198235 & -3753.198283 & -3809.358850 & -3809.358848 \\
\hline & 4 & -3747.523469 & -3753.198235 & -3753.198283 & -3809.358850 & -3809.358848 \\
\hline & 5 & -3747.517232 & -3753.191638 & -3753.191587 & -3809.352165 & -3809.352219 \\
\hline & 6 & -3747.517232 & -3753.191638 & -3753.191587 & -3809.352165 & -3809.352219 \\
\hline & 7 & -3747.516366 & -3753.190250 & -3753.190147 & -3809.351139 & -3809.351334 \\
\hline & 8 & -3747.516366 & -3753.190250 & -3753.190147 & -3809.351139 & -3809.351334 \\
\hline & 9 & -3747.503295 & -3753.176064 & -3753.176101 & -3809.338882 & -3809.338888 \\
\hline \multirow{20}{*}{$\mathrm{Cu}(\mathrm{dtc}) 2$} & 10 & -3747.503295 & -3753.176064 & -3753.176101 & -3809.338882 & -3809.338888 \\
\hline & 11 & -3747.370089 & -3753.131702 & -3753.099658 & -3809.317345 & -3809.269224 \\
\hline & 12 & -3747.370089 & -3753.131702 & -3753.099658 & -3809.317345 & -3809.269224 \\
\hline & 13 & -3745.700664 & -3751.540578 & -3751.540547 & -3807.704714 & -3807.704828 \\
\hline & 14 & -3745.700664 & -3751.540578 & -3751.540547 & -3807.704714 & -3807.704828 \\
\hline & 15 & -3745.681304 & -3751.534824 & -3751.534367 & -3807.699718 & -3807.699748 \\
\hline & 16 & -3745.681304 & -3751.534824 & -3751.534367 & -3807.699718 & -3807.699748 \\
\hline & 17 & -3745.661938 & -3751.499086 & -3751.499140 & -3807.653480 & -3807.653377 \\
\hline & 18 & -3745.661938 & -3751.499086 & -3751.499140 & -3807.653480 & -3807.653377 \\
\hline & 19 & -3745.645127 & -3751.490468 & -3751.490587 & -3807.647169 & -3807.646954 \\
\hline & 20 & -3745.645127 & -3751.490468 & -3751.490587 & -3807.647169 & -3807.646954 \\
\hline & 1 & -2130.049770 & -2134.253803 & -2134.252286 & -2168.995662 & -2168.997655 \\
\hline & 2 & -2130.049770 & -2134.253803 & -2134.252286 & -2168.995662 & -2168.997655 \\
\hline & 3 & -2129.975558 & -2134.178476 & -2134.178498 & -2168.936291 & -2168.936307 \\
\hline & 4 & -2129.975558 & -2134.178476 & -2134.178498 & -2168.936291 & -2168.936307 \\
\hline & 5 & -2129.957146 & -2134.161043 & -2134.161393 & -2168.920035 & -2168.919920 \\
\hline & 6 & -2129.957146 & -2134.161043 & -2134.161393 & -2168.920035 & -2168.919920 \\
\hline & 7 & -2129.954265 & -2134.157989 & -2134.158574 & -2168.917437 & -2168.917914 \\
\hline & 8 & -2129.954265 & -2134.157989 & -2134.158574 & -2168.917437 & -2168.917914 \\
\hline & 9 & -2129.930400 & -2134.130657 & -2134.131104 & -2168.898631 & -2168.898190 \\
\hline \multirow{31}{*}[\mathrm{Ni}(\text{cyclam})]{$^{+}$} & 10 & -2129.930400 & -2134.130657 & -2134.131104 & -2168.898631 & -2168.898190 \\
\hline & 11 & -2129.549747 & -2133.868835 & -2133.859898 & -2168.599002 & -2168.587384 \\
\hline & 12 & -2129.549747 & -2133.868835 & -2133.859898 & -2168.599002 & -2168.587384 \\
\hline & 13 & -2129.124329 & -2133.421933 & -2133.421680 & -2168.146575 & -2168.147852 \\
\hline & 14 & -2129.124329 & -2133.421933 & -2133.421680 & -2168.146575 & -2168.147852 \\
\hline & 15 & -2129.116826 & -2133.407809 & -2133.406428 & -2168.138817 & -2168.139052 \\
\hline & 16 & -2129.116826 & -2133.407809 & -2133.406428 & -2168.138817 & -2168.139052 \\
\hline & 17 & -2129.113590 & -2133.388513 & -2133.389612 & -2168.124227 & -2168.125199 \\
\hline & 18 & -2129.113590 & -2133.388513 & -2133.389612 & -2168.124227 & -2168.125199 \\
\hline & 19 & -2129.111173 & -2133.385218 & -2133.386388 & -2168.121202 & -2168.122209 \\
\hline & 20 & -2129.111173 & -2133.385218 & -2133.386388 & -2168.121202 & -2168.122209 \\
\hline & 1 & -1858.360223 & -1860.855071 & -1860.852732 & -1885.200367 & -1885.197439 \\
\hline & 2 & -1858.360223 & -1860.855071 & -1860.852732 & -1885.200367 & -1885.197439 \\
\hline & 3 & -1858.281614 & -1860.774365 & -1860.774268 & -1885.116115 & -1885.116089 \\
\hline & 4 & -1858.281614 & -1860.774365 & -1860.774268 & -1885.116115 & -1885.116089 \\
\hline & 5 & -1858.278505 & -1860.771327 & -1860.771179 & -1885.113068 & -1885.112966 \\
\hline & 6 & -1858.278505 & -1860.771327 & -1860.771179 & -1885.113068 & -1885.112966 \\
\hline & 7 & -1858.271511 & -1860.761599 & -1860.761863 & -1885.104212 & -1885.104574 \\
\hline & 8 & -1858.271511 & -1860.761599 & -1860.761863 & -1885.104212 & -1885.104574 \\
\hline & 9 & -1858.266096 & -1860.756243 & -1860.756421 & -1885.098838 & -1885.099123 \\
\hline & 10 & -1858.266096 & -1860.756243 & -1860.756421 & -1885.098838 & -1885.099123 \\
\hline & 11 & -1858.192762 & -1860.699038 & -1860.698024 & -1885.047712 & -1885.048160 \\
\hline & 12 & -1858.192762 & -1860.699038 & -1860.698024 & -1885.047712 & -1885.048160 \\
\hline & 13 & -1857.781283 & -1860.325537 & -1860.333002 & -1884.692090 & -1884.695753 \\
\hline & 14 & -1857.781283 & -1860.325537 & -1860.333002 & -1884.692090 & -1884.695753 \\
\hline & 15 & -1857.767523 & -1860.318272 & -1860.318274 & -1884.690263 & -1884.690262 \\
\hline & 16 & -1857.767523 & -1860.318272 & -1860.318274 & -1884.690263 & -1884.690262 \\
\hline & 17 & -1857.762337 & -1860.313085 & -1860.313087 & -1884.685074 & -1884.685074 \\
\hline & 18 & -1857.762337 & -1860.313085 & -1860.313087 & -1884.685074 & -1884.685074 \\
\hline & 19 & -1857.731346 & -1860.290974 & -1860.282343 & -1884.649542 & -1884.646179 \\
\hline & 20 & -1857.731346 & -1860.290974 & -1860.282343 & -1884.649542 & -1884.646179 \\
\hline
\end{tabular}


S-36

Table S5.6. Absolute energies (in hartree) for $\mathrm{d}^{7}$ configurations at CASCI, MC-PDFT and XMSPDFT levels with CASCI scheme.

\begin{tabular}{|c|c|c|c|c|c|c|}
\hline Molecule & $\begin{array}{l}\text { Spin- } \\
\text { orbit } \\
\text { state } \\
\end{array}$ & CASCI $(1,10)$ & $\begin{array}{c}\mathrm{MC}(1,10)- \\
\mathrm{PDFT} \\
(\mathrm{tPBE})\end{array}$ & $\begin{array}{c}\text { XMS }(1,10)- \\
\text { PDFT } \\
(\mathrm{tPBE})\end{array}$ & $\begin{array}{c}\mathrm{MC}(1,10)- \\
\text { PDFT } \\
\text { (tPBE-HLE) }\end{array}$ & $\begin{array}{c}\text { XMS }(1,10)- \\
\text { PDFT } \\
\text { (tPBE-HLE) }\end{array}$ \\
\hline \multirow[t]{20}{*}{ Co(acacen) } & 1 & -2116.183500 & -2120.722849 & -2120.723209 & -2157.304678 & -2157.305124 \\
\hline & 2 & -2116.183500 & -2120.722849 & -2120.723209 & -2157.304678 & -2157.305124 \\
\hline & 3 & -2116.176865 & -2120.714760 & -2120.714878 & -2157.286970 & -2157.287058 \\
\hline & 4 & -2116.176865 & -2120.714760 & -2120.714878 & -2157.286970 & -2157.287058 \\
\hline & 5 & -2116.168694 & -2120.703470 & -2120.703508 & -2157.278171 & -2157.278150 \\
\hline & 6 & -2116.168694 & -2120.703470 & -2120.703508 & -2157.278171 & -2157.278150 \\
\hline & 7 & -2116.129477 & -2120.667916 & -2120.667948 & -2157.234217 & -2157.234321 \\
\hline & 8 & -2116.129477 & -2120.667916 & -2120.667948 & -2157.234217 & -2157.234321 \\
\hline & 9 & -2116.073638 & -2120.610038 & -2120.610246 & -2157.198046 & -2157.200466 \\
\hline & 10 & -2116.073638 & -2120.610038 & -2120.610246 & -2157.198046 & -2157.200466 \\
\hline & 11 & -2116.068549 & -2120.604077 & -2120.607029 & -2157.194034 & -2157.196173 \\
\hline & 12 & -2116.068549 & -2120.604077 & -2120.607029 & -2157.194034 & -2157.196173 \\
\hline & 13 & -2116.064281 & -2120.596533 & -2120.602778 & -2157.183767 & -2157.192799 \\
\hline & 14 & -2116.064281 & -2120.596533 & -2120.602778 & -2157.183767 & -2157.192799 \\
\hline & 15 & -2116.056082 & -2120.595040 & -2120.594506 & -2157.183102 & -2157.184069 \\
\hline & 16 & -2116.056082 & -2120.595040 & -2120.594506 & -2157.183102 & -2157.184069 \\
\hline & 17 & -2116.054325 & -2120.591181 & -2120.591767 & -2157.182835 & -2157.182453 \\
\hline & 18 & -2116.054325 & -2120.591181 & -2120.591767 & -2157.182835 & -2157.182453 \\
\hline & 19 & -2116.052212 & -2120.590198 & -2120.590153 & -2157.169223 & -2157.174011 \\
\hline & 20 & -2116.052212 & -2120.590198 & -2120.590153 & -2157.169223 & -2157.174011 \\
\hline \multirow[t]{20}{*}{$\mathrm{Co}(\mathrm{sacsac})_{2}$} & 1 & -3373.662884 & -3378.766517 & -3378.765769 & -3429.529505 & -3429.528468 \\
\hline & 2 & -3373.662884 & -3378.766517 & -3378.765769 & -3429.529505 & -3429.528468 \\
\hline & 3 & -3373.654695 & -3378.757114 & -3378.757549 & -3429.514355 & -3429.514882 \\
\hline & 4 & -3373.654695 & -3378.757114 & -3378.757549 & -3429.514355 & -3429.514882 \\
\hline & 5 & -3373.639184 & -3378.736563 & -3378.736842 & -3429.496796 & -3429.497146 \\
\hline & 6 & -3373.639184 & -3378.736563 & -3378.736842 & -3429.496796 & -3429.497146 \\
\hline & 7 & -3373.597061 & -3378.698353 & -3378.709943 & -3429.453510 & -3429.465285 \\
\hline & 8 & -3373.597061 & -3378.698353 & -3378.709943 & -3429.453510 & -3429.465285 \\
\hline & 9 & -3373.543995 & -3378.662364 & -3378.660751 & -3429.425339 & -3429.421879 \\
\hline & 10 & -3373.543995 & -3378.662364 & -3378.660751 & -3429.425339 & -3429.421879 \\
\hline & 11 & -3373.541614 & -3378.650971 & -3378.650628 & -3429.422494 & -3429.420585 \\
\hline & 12 & -3373.541614 & -3378.650971 & -3378.650628 & -3429.422494 & -3429.420585 \\
\hline & 13 & -3373.538862 & -3378.646087 & -3378.646084 & -3429.419613 & -3429.416399 \\
\hline & 14 & -3373.538862 & -3378.646087 & -3378.646084 & -3429.419613 & -3429.416399 \\
\hline & 15 & -3373.528052 & -3378.637548 & -3378.642798 & -3429.408322 & -3429.415040 \\
\hline & 16 & -3373.528052 & -3378.637548 & -3378.642798 & -3429.408322 & -3429.415040 \\
\hline & 17 & -3373.527092 & -3378.632236 & -3378.632335 & -3429.398984 & -3429.399218 \\
\hline & 18 & -3373.527092 & -3378.632236 & -3378.632335 & -3429.398984 & -3429.399218 \\
\hline & 19 & -3373.524513 & -3378.630600 & -3378.631177 & -3429.392269 & -3429.394224 \\
\hline & 20 & -3373.524513 & -3378.630600 & -3378.631177 & -3429.392269 & -3429.394224 \\
\hline \multirow[t]{3}{*}{ Co(tacacen) } & 1 & -2763.526386 & -2768.527985 & -2768.528424 & -2813.141930 & -2813.140718 \\
\hline & 2 & -2763.526386 & -2768.527985 & -2768.528424 & -2813.141930 & -2813.140718 \\
\hline & 3 & -2763.517834 & -2768.519592 & -2768.519977 & -2813.127899 & -2813.129163 \\
\hline
\end{tabular}




\begin{tabular}{|c|c|c|c|c|c|c|}
\hline & 4 & -2763.517834 & -2768.519592 & -2768.519977 & -2813.127899 & -2813.129163 \\
\hline & 5 & -2763.504912 & -2768.502034 & -2768.502073 & -2813.112719 & -2813.112764 \\
\hline & 6 & -2763.504912 & -2768.502034 & -2768.502073 & -2813.112719 & -2813.112764 \\
\hline & 7 & -2763.457947 & -2768.463920 & -2768.464041 & -2813.069343 & -2813.070366 \\
\hline & 8 & -2763.457947 & -2768.463920 & -2768.464041 & -2813.069343 & -2813.070366 \\
\hline & 9 & -2763.361212 & -2768.375570 & -2768.377455 & -2813.004562 & -2813.005594 \\
\hline & 10 & -2763.361212 & -2768.375570 & -2768.377455 & -2813.004562 & -2813.005594 \\
\hline & 11 & -2763.353907 & -2768.373133 & -2768.371779 & -2813.003807 & -2813.001728 \\
\hline & 12 & -2763.353907 & -2768.373133 & -2768.371779 & -2813.003807 & -2813.001728 \\
\hline & 13 & -2763.350973 & -2768.367467 & -2768.367924 & -2812.994540 & -2812.994085 \\
\hline & 14 & -2763.350973 & -2768.367467 & -2768.367924 & -2812.994540 & -2812.994085 \\
\hline & 15 & -2763.346017 & -2768.366547 & -2768.365682 & -2812.992833 & -2812.992500 \\
\hline & 16 & -2763.346017 & -2768.366547 & -2768.365682 & -2812.992833 & -2812.992500 \\
\hline & 17 & -2763.345445 & -2768.364029 & -2768.363428 & -2812.991894 & -2812.990931 \\
\hline & 18 & -2763.345445 & -2768.364029 & -2768.363428 & -2812.991894 & -2812.990931 \\
\hline & 19 & -2763.344883 & -2768.362056 & -2768.361687 & -2812.987791 & -2812.987709 \\
\hline & 20 & -2763.344883 & -2768.362056 & -2768.361687 & -2812.987791 & -2812.987709 \\
\hline & 1 & -3635.017370 & -3640.526529 & -3640.525609 & -3694.958165 & -3694.956619 \\
\hline & 2 & -3635.017370 & -3640.526529 & -3640.525609 & -3694.958165 & -3694.956619 \\
\hline & 3 & -3635.006934 & -3640.514402 & -3640.514448 & -3694.941926 & -3694.941741 \\
\hline & 4 & -3635.006934 & -3640.514402 & -3640.514448 & -3694.941926 & -3694.941741 \\
\hline & 5 & -3635.000717 & -3640.507497 & -3640.507395 & -3694.935700 & -3694.935720 \\
\hline & 6 & -3635.000717 & -3640.507497 & -3640.507395 & -3694.935700 & -3694.935720 \\
\hline & 7 & -3634.957850 & -3640.468939 & -3640.466439 & -3694.896536 & -3694.892944 \\
\hline & 8 & -3634.957850 & -3640.468939 & -3640.466439 & -3694.896536 & -3694.892944 \\
\hline & 9 & -3634.869598 & -3640.407515 & -3640.407435 & -3694.841453 & -3694.841335 \\
\hline & 10 & -3634.869598 & -3640.407515 & -3640.407435 & -3694.841453 & -3694.841335 \\
\hline & 11 & -3634.868191 & -3640.374711 & -3640.393056 & -3694.812536 & -3694.821662 \\
\hline & 12 & -3634.868191 & -3640.374711 & -3640.393056 & -3694.812536 & -3694.821662 \\
\hline & 13 & -3634.863543 & -3640.367462 & -3640.367464 & -3694.812139 & -3694.810071 \\
\hline & 14 & -3634.863543 & -3640.367462 & -3640.367464 & -3694.812139 & -3694.810071 \\
\hline & 15 & -3634.862918 & -3640.365499 & -3640.365815 & -3694.807697 & -3694.809554 \\
\hline & 16 & -3634.862918 & -3640.365499 & -3640.365815 & -3694.807697 & -3694.809554 \\
\hline & 17 & -3634.852600 & -3640.361045 & -3640.360954 & -3694.806418 & -3694.794040 \\
\hline & 18 & -3634.852600 & -3640.361045 & -3640.360954 & -3694.806418 & -3694.794040 \\
\hline & 19 & -3634.817801 & -3640.333212 & -3640.315632 & -3694.750720 & -3694.742711 \\
\hline & 20 & -3634.817801 & -3640.333212 & -3640.315632 & -3694.750720 & -3694.742711 \\
\hline
\end{tabular}


Table S5.7. Absolute energies (in hartree) for $\mathrm{d}^{5}$ configurations at CASCI, MC-PDFT and XMSPDFT levels with CASCI scheme.

\begin{tabular}{|c|c|c|c|c|c|c|}
\hline Molecule & $\begin{array}{l}\text { Spin-orbit } \\
\text { state }\end{array}$ & $\operatorname{CASCI}(N, 10)$ & $\begin{array}{c}\operatorname{MC}(N, 10)- \\
\text { PDFT (tPBE) }\end{array}$ & $\begin{array}{l}\text { XMS }(N, 10)- \\
\text { PDFT (tPBE) }\end{array}$ & $\begin{array}{c}\mathrm{MC}(N, 10)-\mathrm{PDFT} \\
(\mathrm{tPBE}-\mathrm{HLE})\end{array}$ & $\begin{array}{l}\text { XMS }(N, 10)-P D F T \\
\text { (tPBE-HLE) }\end{array}$ \\
\hline \multirow{29}{*}[\mathrm{Fe}(\mathrm{bpy})3]{$^{3+}$} & 1 & -2748.570619 & -2756.845566 & -2756.845514 & -2816.518496 & -2816.518414 \\
\hline & 2 & -2748.570619 & -2756.845566 & -2756.845514 & -2816.518496 & -2816.518414 \\
\hline & 3 & -2748.564659 & -2756.837977 & -2756.837966 & -2816.510812 & -2816.510708 \\
\hline & 4 & -2748.564659 & -2756.837977 & -2756.837966 & -2816.510812 & -2816.510708 \\
\hline & 5 & -2748.563174 & -2756.836369 & -2756.836357 & -2816.509197 & -2816.509090 \\
\hline & 6 & -2748.563174 & -2756.836369 & -2756.836357 & -2816.509197 & -2816.509090 \\
\hline & 7 & -2748.472778 & -2756.732399 & -2756.732494 & -2816.407378 & -2816.405548 \\
\hline & 8 & -2748.472778 & -2756.732399 & -2756.732494 & -2816.407378 & -2816.405548 \\
\hline & 9 & -2748.471767 & -2756.731993 & -2756.731906 & -2816.406403 & -2816.403399 \\
\hline & 10 & -2748.471767 & -2756.731993 & -2756.731906 & -2816.406403 & -2816.403399 \\
\hline & 11 & -2748.470592 & -2756.730459 & -2756.730120 & -2816.403779 & -2816.402202 \\
\hline & 12 & -2748.470592 & -2756.730459 & -2756.730120 & -2816.403779 & -2816.402202 \\
\hline & 13 & -2748.463506 & -2756.726621 & -2756.726163 & -2816.397490 & -2816.396935 \\
\hline & 14 & -2748.463506 & -2756.726621 & -2756.726163 & -2816.397490 & -2816.396935 \\
\hline & 15 & -2748.455447 & -2756.722578 & -2756.720004 & -2816.396105 & -2816.390737 \\
\hline & 16 & -2748.455447 & -2756.722578 & -2756.720004 & -2816.396105 & -2816.390737 \\
\hline & 17 & -2748.455129 & -2756.722241 & -2756.716204 & -2816.395812 & -2816.387017 \\
\hline & 18 & -2748.455129 & -2756.722241 & -2756.716204 & -2816.395812 & -2816.387017 \\
\hline & 19 & -2748.452078 & -2756.719826 & -2756.715805 & -2816.390821 & -2816.386328 \\
\hline & 20 & -2748.452078 & -2756.719826 & -2756.715805 & -2816.390821 & -2816.386328 \\
\hline & 1 & -2442.650331 & -2449.447447 & -2449.447035 & -2499.322553 & -2499.323728 \\
\hline & 2 & -2442.650331 & -2449.447447 & -2449.447035 & -2499.322553 & -2499.323728 \\
\hline & 3 & -2442.638286 & -2449.433737 & -2449.433604 & -2499.310292 & -2499.310958 \\
\hline & 4 & -2442.638286 & -2449.433737 & -2449.433604 & -2499.310292 & -2499.310958 \\
\hline & 5 & -2442.632015 & -2449.428176 & -2449.427509 & -2499.303810 & -2499.304857 \\
\hline & 6 & -2442.632015 & -2449.428176 & -2449.427509 & -2499.303810 & -2499.304857 \\
\hline & 7 & -2442.534545 & -2449.341015 & -2449.340731 & -2499.228185 & -2499.227469 \\
\hline & 8 & -2442.534545 & -2449.341015 & -2449.340731 & -2499.228185 & -2499.227469 \\
\hline & 9 & -2442.527570 & -2449.327129 & -2449.329591 & -2499.209464 & -2499.216164 \\
\hline$\left[\mathrm{Fe}\left(\mathrm{N}_{3}\right)(\mathrm{Me}\right.$ & 10 & -2442.527570 & -2449.327129 & -2449.329591 & -2499.209464 & -2499.216164 \\
\hline \multirow{18}{*}{$\mathrm{Py}_{2}$ tacn) $]^{2+}$} & 11 & -2442.523567 & -2449.326251 & -2449.327817 & -2499.206314 & -2499.214511 \\
\hline & 12 & -2442.523567 & -2449.326251 & -2449.327817 & -2499.206314 & -2499.214511 \\
\hline & 13 & -2442.520872 & -2449.323601 & -2449.323074 & -2499.205593 & -2499.211092 \\
\hline & 14 & -2442.520872 & -2449.323601 & -2449.323074 & -2499.205593 & -2499.211092 \\
\hline & 15 & -2442.518804 & -2449.321941 & -2449.319358 & -2499.203657 & -2499.206662 \\
\hline & 16 & -2442.518804 & -2449.321941 & -2449.319358 & -2499.203657 & -2499.206662 \\
\hline & 17 & -2442.506953 & -2449.313340 & -2449.309863 & -2499.192594 & -2499.197334 \\
\hline & 18 & -2442.506953 & -2449.313340 & -2449.309863 & -2499.192594 & -2499.197334 \\
\hline & 19 & -2442.504062 & -2449.310996 & -2449.306584 & -2499.187669 & -2499.184934 \\
\hline & 20 & -2442.504062 & -2449.310996 & -2449.306584 & -2499.187669 & -2499.184934 \\
\hline & 1 & -3059.607926 & -3066.636002 & -3066.636093 & -3123.462334 & -3123.462356 \\
\hline & 2 & -3059.607926 & -3066.636002 & -3066.636093 & -3123.462334 & -3123.462356 \\
\hline & 3 & -3059.579705 & -3066.604275 & -3066.611065 & -3123.434601 & -3123.450320 \\
\hline & 4 & -3059.579705 & -3066.604275 & -3066.611065 & -3123.434601 & -3123.450320 \\
\hline & 5 & -3059.573797 & -3066.598642 & -3066.598898 & -3123.429327 & -3123.429596 \\
\hline & 6 & -3059.573797 & -3066.598642 & -3066.598898 & -3123.429327 & -3123.429596 \\
\hline & 7 & -3059.478171 & -3066.511726 & -3066.521009 & -3123.342249 & -3123.353045 \\
\hline & 8 & -3059.478171 & -3066.511726 & -3066.521009 & -3123.342249 & -3123.353045 \\
\hline \multirow[t]{9}{*}[\mathrm{Fe}(\mathrm{PyMS})2]{$^{+}$} & 9 & -3059.476042 & -3066.511056 & -3066.506271 & -3123.341261 & -3123.342210 \\
\hline & 10 & -3059.476042 & -3066.511056 & -3066.506271 & -3123.341261 & -3123.342210 \\
\hline & 11 & -3059.474085 & -3066.507773 & -3066.504840 & -3123.340076 & -3123.341017 \\
\hline & 12 & -3059.474085 & -3066.507773 & -3066.504840 & -3123.340076 & -3123.341017 \\
\hline & 13 & -3059.465410 & -3066.499791 & -3066.498830 & -3123.336823 & -3123.337659 \\
\hline & 14 & -3059.465410 & -3066.499791 & -3066.498830 & -3123.336823 & -3123.337659 \\
\hline & 15 & -3059.450367 & -3066.487790 & -3066.478963 & -3123.317200 & -3123.314730 \\
\hline & 16 & -3059.450367 & -3066.487790 & -3066.478963 & -3123.317200 & -3123.314730 \\
\hline & 17 & -3059.448260 & -3066.485772 & -3066.477514 & -3123.314504 & -3123.313497 \\
\hline
\end{tabular}




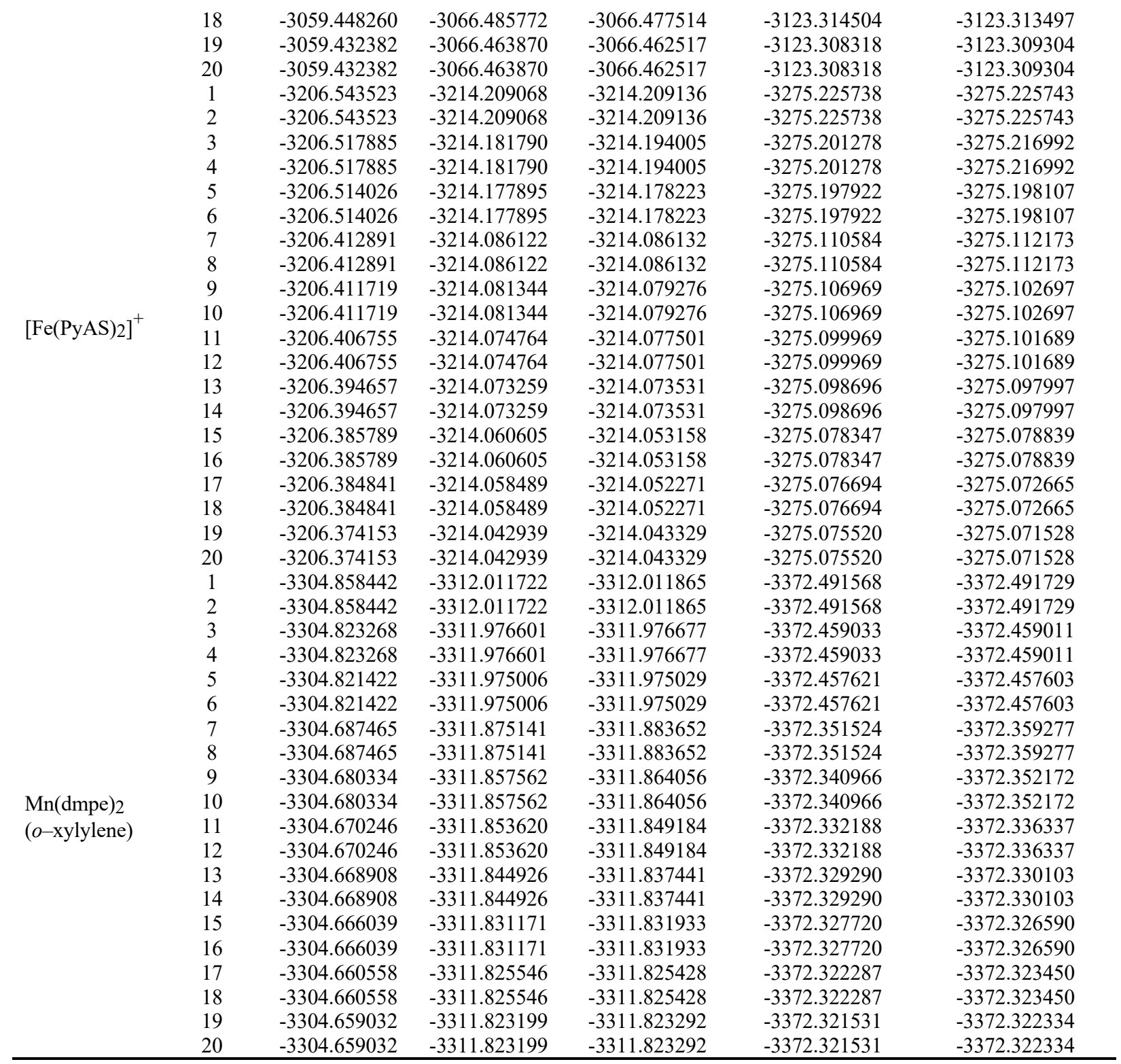


Table S5.8. Absolute energies (in hartree) for $\mathrm{d}^{1}$ configurations at CASCI, MC-PDFT and XMSPDFT levels with CASCI scheme.

\begin{tabular}{|c|c|c|c|c|c|c|}
\hline Molecule & $\begin{array}{l}\text { Spin- } \\
\text { orbit } \\
\text { state }\end{array}$ & $\operatorname{CASCI}(1, R)$ & $\begin{array}{l}\mathrm{MC}(1, R)-\mathrm{PDFT} \\
\quad(\mathrm{tPBE})\end{array}$ & $\begin{array}{l}\text { XMS }(1, R)- \\
\text { PDFT (tPBE) }\end{array}$ & $\begin{array}{c}\mathrm{MC}(1, R)-\mathrm{PDFT} \\
\quad(\mathrm{tPBE}-\mathrm{HLE})\end{array}$ & $\begin{array}{l}\text { XMS }(1, R)- \\
\text { PDFT } \\
\text { (tPBE-HLE) }\end{array}$ \\
\hline \multirow{20}{*}[\mathrm{MnN}(\mathrm{CN})4]{$^{-}$} & 1 & -1581.342867 & -1584.290903 & -1584.297613 & -1608.966173 & -1608.966636 \\
\hline & 2 & -1581.342867 & -1584.290903 & -1584.297613 & -1608.966173 & -1608.966636 \\
\hline & 3 & -1581.202519 & -1584.172950 & -1584.181272 & -1608.847672 & -1608.849684 \\
\hline & 4 & -1581.202519 & -1584.172950 & -1584.181272 & -1608.847672 & -1608.849684 \\
\hline & 5 & -1581.202042 & -1584.172473 & -1584.180558 & -1608.847195 & -1608.845753 \\
\hline & 6 & -1581.202042 & -1584.172473 & -1584.180558 & -1608.847195 & -1608.845753 \\
\hline & 7 & -1581.166170 & -1584.132907 & -1584.125906 & -1608.806103 & -1608.803130 \\
\hline & 8 & -1581.166170 & -1584.132907 & -1584.125906 & -1608.806103 & -1608.803130 \\
\hline & 9 & -1581.145838 & -1584.132274 & -1584.125036 & -1608.796204 & -1608.794794 \\
\hline & 10 & -1581.145838 & -1584.132274 & -1584.125036 & -1608.796204 & -1608.794794 \\
\hline & 11 & -1581.145202 & -1584.114672 & -1584.105892 & -1608.795566 & -1608.786641 \\
\hline & 12 & -1581.145202 & -1584.114672 & -1584.105892 & -1608.795566 & -1608.786641 \\
\hline & 13 & -1581.124441 & -1584.089383 & -1584.089383 & -1608.772859 & -1608.772858 \\
\hline & 14 & -1581.124441 & -1584.089383 & -1584.089383 & -1608.772859 & -1608.772858 \\
\hline & 15 & -1581.124008 & -1584.087073 & -1584.087073 & -1608.769926 & -1608.769925 \\
\hline & 16 & -1581.124008 & -1584.087073 & -1584.087073 & -1608.769926 & -1608.769925 \\
\hline & 17 & -1581.103786 & -1584.076514 & -1584.078371 & -1608.746063 & -1608.748484 \\
\hline & 18 & -1581.103786 & -1584.076514 & -1584.078371 & -1608.746063 & -1608.748484 \\
\hline & 19 & -1581.078154 & -1584.070032 & -1584.067319 & -1608.740451 & -1608.736938 \\
\hline & 20 & -1581.078154 & -1584.070032 & -1584.067319 & -1608.740451 & -1608.736938 \\
\hline \multirow{20}{*}{$\begin{array}{c}{[\mathrm{MnN}(\text { cyclam })} \\
\left.\left(\mathrm{NCCH}_{3}\right)\right]^{3+}\end{array}$} & 1 & -1953.829698 & -1958.812349 & -1958.820978 & -1996.361939 & -1996.362908 \\
\hline & 2 & -1953.829698 & -1958.812349 & -1958.820978 & -1996.361939 & -1996.362908 \\
\hline & 3 & -1953.695120 & -1958.700963 & -1958.708051 & -1996.246932 & -1996.249853 \\
\hline & 4 & -1953.695120 & -1958.700963 & -1958.708051 & -1996.246932 & -1996.249853 \\
\hline & 5 & -1953.693800 & -1958.700111 & -1958.705295 & -1996.246118 & -1996.248981 \\
\hline & 6 & -1953.693800 & -1958.700111 & -1958.705295 & -1996.246118 & -1996.248981 \\
\hline & 7 & -1953.665977 & -1958.660239 & -1958.644381 & -1996.208453 & -1996.204425 \\
\hline & 8 & -1953.665977 & -1958.660239 & -1958.644381 & -1996.208453 & -1996.204425 \\
\hline & 9 & -1953.633358 & -1958.659617 & -1958.639731 & -1996.195865 & -1996.187732 \\
\hline & 10 & -1953.633358 & -1958.659617 & -1958.639731 & -1996.195865 & -1996.187732 \\
\hline & 11 & -1953.632244 & -1958.650244 & -1958.638530 & -1996.195117 & -1996.184735 \\
\hline & 12 & -1953.632244 & -1958.650244 & -1958.638530 & -1996.195117 & -1996.184735 \\
\hline & 13 & -1953.624738 & -1958.625178 & -1958.625348 & -1996.178235 & -1996.178208 \\
\hline & 14 & -1953.624738 & -1958.625178 & -1958.625348 & -1996.178235 & -1996.178208 \\
\hline & 15 & -1953.623934 & -1958.622653 & -1958.622401 & -1996.174970 & -1996.174833 \\
\hline & 16 & -1953.623934 & -1958.622653 & -1958.622401 & -1996.174970 & -1996.174833 \\
\hline & 17 & -1953.603564 & -1958.613269 & -1958.614079 & -1996.154303 & -1996.154398 \\
\hline & 18 & -1953.603564 & -1958.613269 & -1958.614079 & -1996.154303 & -1996.154398 \\
\hline & 19 & -1953.575697 & -1958.605225 & -1958.603556 & -1996.145284 & -1996.144714 \\
\hline & 20 & -1953.575697 & -1958.605225 & -1958.603556 & -1996.145284 & -1996.144714 \\
\hline \multirow{5}{*}{$\mathrm{VO}(\mathrm{acac}) 2$} & 1 & -1710.177874 & -1714.541376 & -1714.541414 & -1747.724523 & -1747.723930 \\
\hline & 2 & -1710.177874 & -1714.541376 & -1714.541414 & -1747.724523 & -1747.723930 \\
\hline & 3 & -1710.101323 & -1714.460100 & -1714.459972 & -1747.657830 & -1747.657962 \\
\hline & 4 & -1710.101323 & -1714.460100 & -1714.459972 & -1747.657830 & -1747.657962 \\
\hline & 5 & -1710.098347 & -1714.446955 & -1714.446955 & -1747.634934 & -1747.634946 \\
\hline
\end{tabular}




\begin{tabular}{ccccccc} 
& 6 & -1710.098347 & -1714.446955 & -1714.446955 & -1747.634934 & -1747.634946 \\
& 7 & -1710.094416 & -1714.443266 & -1714.443321 & -1747.631877 & -1747.632280 \\
& 8 & -1710.094416 & -1714.443266 & -1714.443321 & -1747.631877 & -1747.632280 \\
& 9 & -1710.031112 & -1714.366835 & -1714.366857 & -1747.547249 & -1747.547278 \\
& 10 & -1710.031112 & -1714.366835 & -1714.366857 & -1747.547249 & -1747.547278 \\
& 1 & -1403.237480 & -1405.970771 & -1405.970918 & -1428.766748 & -1428.764866 \\
$\mathrm{VO}\left(\mathrm{H}_{2} \mathrm{O}\right) 5^{2+}$ & 2 & -1403.237480 & -1405.970771 & -1405.970918 & -1428.766748 & -1428.764866 \\
& 3 & -1403.170159 & -1405.900024 & -1405.899765 & -1428.710539 & -1428.710239 \\
& 4 & -1403.170159 & -1405.900024 & -1405.899765 & -1428.710539 & -1428.710239 \\
& 5 & -1403.153040 & -1405.865160 & -1405.865226 & -1428.663940 & -1428.664504 \\
& 6 & -1403.153040 & -1405.865160 & -1405.865226 & -1428.663940 & -1428.664504 \\
& 7 & -1403.150241 & -1405.863514 & -1405.863943 & -1428.662884 & -1428.663245 \\
& 8 & -1403.150241 & -1405.863514 & -1405.863943 & -1428.662884 & -1428.663245 \\
& 9 & -1403.047443 & -1405.754054 & -1405.754973 & -1428.556289 & -1428.558742 \\
& 10 & -1403.047443 & -1405.754054 & -1405.754973 & -1428.556289 & -1428.558742 \\
\hline
\end{tabular}

